\title{
EXTENDED MCKAY CORRESPONDENCE FOR QUOTIENT SURFACE SINGULARITIES
}

\author{
AKIRA ISHII AND IKU NAKAMURA, JULY 27, 2018
}

\begin{abstract}
Let $G$ be a finite subgroup of GL(2) acting on $\mathbf{A}^{2} \backslash\{0\}$ freely. The $G$-orbit Hilbert scheme $G-\operatorname{Hilb}\left(\mathbf{A}^{2}\right)$ is a minimal resolution of the quotient $\mathbf{A}^{2} / G$ by [19]. We determine the generator sheaf of the ideal defining the universal $G$-cluster over $G$-Hilb $\left(\mathbf{A}^{2}\right)$, which somewhat strengthens the well-known McKay correspondence for a finite subgroup of $\operatorname{SL}(2)$. We also study the quiver structure of $G$-Hilb $\left(\mathbf{A}^{2}\right)$ at every $G$-cluster $O_{Z_{y}}=O_{\mathbf{A}^{2}} / I_{y}$ in terms of a collection of sort of minimal

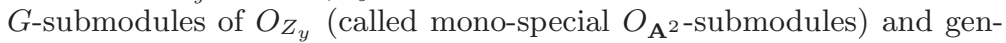
erating $G$-submodules of $I_{y}$.
\end{abstract}

\section{INTRODUCTION.}

For an algebraically closed field $k$, let $G$ be any finite small subgroup of $\operatorname{GL}(2, k)$, that is, a finite subgroup with no pseudo-reflections, or equivalently, a finite subgroup of $\mathrm{GL}(2, k)$ acting on $\mathbf{A}_{k}^{2}$ with the origin the unique fixed point of it. Throughout this article we assume that the characteristic of $k$ is prime to the order $|G|$ of $G$.

The $G$-orbit Hilbert scheme $G$-Hilb $\left(\mathbf{A}_{k}^{2}\right)$ is the scheme parameterizing all the $G$-invariant zero-dimensional subschemes of $\mathbf{A}_{k}^{2}$ of length $|G|$, each with structure sheaf isomorphic to the group algebra $k[G]$ of $G$ as a $k[G]$-module. It is by [19] the minimal resolution of the quotient $\mathbf{A}_{k}^{2} / G$.

For any geometric point $y$ in the exceptional set of the resolution, let $\operatorname{Gen}\left(I_{y}\right)$ be the minimal $k[G]$-(sub)module generating the ideal $I_{y} \subset O_{\mathbf{A}_{k}^{2}}$ corresponding to $y$. One of the purposes of this article is to prove the following:

Theorem 1.1. Let $G$ be a finite small subgroup of $\mathrm{GL}(2, k)$, and $E$ the exceptional set of the minimal resolution $G-\operatorname{Hilb}\left(\mathbf{A}_{k}^{2}\right)$ of $\mathbf{A}_{k}^{2} / G$. Then the generator sheaf of the ideal defining the universal $G$-cluster, that is, the union of all $\operatorname{Gen}\left(I_{y}\right)$ over $E$ is an $O_{F}$-module $G$-isomorphic to

$$
\left(\bigoplus_{\rho \neq \rho_{0}} O_{E(\rho)}(-1) \otimes_{k} \rho\right) \bigoplus O_{F}(-F) \otimes_{k} \rho_{0}
$$

Date: July 27, 2018.

Research was supported in part by the Grants-in-aid (15K04819, S-23224001) for Scientific Research, JSPS.

2000 Mathematics Subject Classification. Primary 14J10, 14J17;Secondary 14D20.

Key words and phrases. Moduli, Hilbert scheme, McKay correspondence. 
where $\rho$ ranges over the set of all non-trivial irreducible representations of $G$, special in the sense of Definition 2.14, $E(\rho)$ is an irreducible component of $E$ associated to $\rho$ and $F$ is the fundamental divisor of $E=F_{\text {red }}$.

See Theorem 3.13 and Corollary 3.14. Our main ingredient for the proof is the derived category method adopted by [6] and [19]. See also [22].

The article also aims at studying the special McKay quiver associated with $G$-Hilb $\left(\mathbf{A}_{k}^{2}\right)$ in terms of its "mono-special $O_{U}$-submodules" of $O_{Z_{y}}$ (see Definition 5.8) and generators of $I_{y}$ at every $G$-cluster $O_{Z_{y}}=O_{U} / I_{y}$.

Here is an outline of the article. In Section 2, we recall reflexive modules and full modules. In Section 3, we review local McKay correspondence for two-dimensional quotient singularities from [19]. Then we formulate and prove its global version, global in the sense that it describes a family of the local versions over the exceptional set (Theorem 1.1). In Section 4, we discuss the connection between tensor product with the natural representation, extensions of the sheaf $O_{Z_{y}}$, and cup products of Ext groups. As an application, we prove Theorem 4.8.

In Section 5, we study the semi-orthogonal projection of $O_{0} \otimes_{k} \rho$ to the essential image of $D^{b}\left(\mathrm{Coh} G\right.$-Hilb $\left.\left(\mathbf{A}^{2}\right)\right)$ for every special representation of $G$. For every quotient surface singularity arising from a subgroup of GL(2), we modify the notion of socles of $O_{Z_{y}}$ so as to make more coherent to analysis of exceptional sets. This is done in Theorem 5.7, where mono-special $O_{U^{-}}$ submodules of $O_{Z_{y}}$ are introduced.

In Section 6, we recall from [7] and [29] the special McKay quiver, which describes the reconstruction algebra of the singularity $X$. We then generalize Theorem 4.8 to every (small) finite subgroup of $\mathrm{GL}(2, k)$. This describes a precise connection between a mono-special $O_{U}$-submodule $V(\sigma)$ of $O_{Z_{y}}$ and a generator $G$-submodule $W(\rho) \subset \operatorname{Gen}\left(I_{y}\right)$ for every individual $G$-cluster $Z_{y}$ and every pair $(\sigma, \rho)$ of special representations. Finally we explain all of the above for two examples, a quotient singularity arising from a cyclic group $\left\langle\frac{1}{12}(1,5)\right\rangle$ of order 12 and the binary dihedral case $D_{5}$.

We are very grateful to John McKay for his encouragement and interest in the subject. We are very grateful also to Alastair Craw for his encouragement and suggestion of better terminology.

\section{ReFleXiVe MOdUles AND TORSION FREE PULLBACKS}

\subsection{Derived category.}

Definition 2.1. Let $X$ be a scheme over a fixed field $k$. Let $C o h(X)$ be the category of coherent $O_{X}$-modules and $K(\operatorname{Coh}(X))$ the category consisting of the bounded complexes of objects and morphisms in $C o h(X)$.

We define a morphism $f: P^{\bullet} \rightarrow Q^{\bullet}$ in $K(\operatorname{Coh}(X))$ to be a quasiisomorphism if $f$ induces an isomorphism on cohomology.

The derived category $D(X)=D(\operatorname{Coh}(X))$ is the localization of $K(\operatorname{Coh}(X))$ at Qis (the monoid of quasi-isomorphisms.), that is, it is the category $K(\operatorname{Coh}(X))$ modulo equivalence defined by Qis. See [14, Def., pp. 49-50]. 
Similarly we define $\operatorname{Coh}_{c}(X)$ to be the category of coherent $O_{X}$-modules with complete supports, $K\left(\operatorname{Coh}_{c}(X)\right)$ the category consisting of bounded complexes of objects and morphisms in $\operatorname{Coh}_{c}(X)$, and $D_{c}(X)$ the derived category of $K\left(\operatorname{Coh}_{c}(X)\right)$.

2.2. Reflexive $O_{X}$-modules and full $O_{Y}$-modules. This subsection is taken from [2], [9], and [33]. Let $Z$ be a scheme of finite type over $k, \mathcal{F}$ a coherent $O_{Z}$-module on $Z$. Then $\mathcal{F}$ is defined to be a reflexive $O_{Z}$-module iff $\mathcal{F}^{\vee \vee} \simeq \mathcal{F}$, where $\mathcal{F}^{\vee}=\mathcal{H o m}_{O_{Z}}\left(\mathcal{F}, O_{Z}\right)$.

Lemma 2.2. Let $Z$ be a normal surface, $Z^{\prime}=Z \backslash \operatorname{Sing}(Z)$ and $i: Z^{\prime} \hookrightarrow Z$ the inclusion. The following is true.

(1) any torsion free module over a discrete valuation ring is free, hence, any torsion free sheaf on a nonsingular curve is locally free.

(2) if $Z$ is nonsingular, any reflexive $O_{Z}$-module is locally free,

(3) if $\mathcal{F}$ is a reflexive $O_{Z}$-module, then $i_{*}\left(i^{*} \mathcal{F}\right)=\mathcal{F}$, and it is uniquely determined by its restriction to $Z^{\prime}$,

(4) if $\mathcal{F}$ is a locally free $O_{Z^{\prime}}$-module, then $i_{*}(\mathcal{F})$ is a reflexive $O_{Z}$-module,

(5) $\mathcal{G}^{\vee}$ is reflexive for any finite $O_{Z}$-module $\mathcal{G}$.

Proof. See [16, Corollary 1.4] for the parts (1) and (2). See [16, Proposition 1.6] for the part (3). See [16, Corollary 1.2] for the part (5). The part (4) is proved as follows. Let $\mathcal{G}=i_{*}(\mathcal{F})$. Since $\mathcal{F}=i^{*}(\mathcal{G})$ is locally free on $Z$, we have $\mathcal{G} \simeq i_{*}\left(i^{*} \mathcal{G}\right) \simeq i_{*}\left(i^{*}\left(\mathcal{G}^{\vee \vee}\right)\right) \supset \mathcal{G}^{\vee \vee}$, from which (4) follows.

The following summarizes [8, Lemma 2,1, 2.2].

Lemma 2.3. Let $X$ be an affine normal surface with rational singularities and $f: Y \rightarrow X$ the minimal resolution of $X$.

(1) Let $M$ be a reflexive $O_{X}$-module, $\mathcal{M}$ the torsion free pullback of $M$ to $Y$, that is, $\mathcal{M}=f^{*} M / O_{Y}$-torsions. Then $f_{*}(\mathcal{M})=M$, and

(i) $\mathcal{M}$ is locally free,

(ii) $\mathcal{M}$ is generated by global sections,

(iii) $R^{1} f_{*}\left(\mathcal{M}^{\vee} \otimes_{O_{Y}} \omega_{Y}\right)=0$.

(2) Conversely if a coherent $O_{Y}$-module $\mathcal{M}$ satisfies (i) and (iii), then $M:=f_{*}(\mathcal{M})$ is a reflexive $O_{X}$-module.

Definition 2.4. We call an $O_{Y}$-module $\mathcal{M}$ a full $O_{Y}$-module (or simply a full sheaf following [8]) if the conditions (i)-(iii) are satisfied.

The following is a corollary of Lemma 2.3 (and Lemma 2.2):

Corollary 2.5. Under the same notation as in Lemma 2.3, there is a bijective correspondence between the following sets

(i) the set of (indecomposable) reflexive $O_{X}$-modules $M$,

(ii) the set of (indecomposable) full $O_{Y}$-modules $\mathcal{M}$.

Corollary 2.6. Under the same notation as in Lemma 2.3, every full $O_{Y^{-}}$ module $\mathcal{M}$ is determined by its restriction to $Y \backslash f^{-1}(\operatorname{Sing}(X))$. 
Proof. Clear from Lemma 2.2 (3).

Corollary 2.7. The invertible sheaf $K_{Y}$ is a full sheaf.

Proof. Clear from Lemma 2.3 (2) and Corollary 2.6.

2.3. The minimal resolution $Y$ of the quotient $X=U / G$. Let $k$ be any algebraically closed field of any characteristic, and $G$ a finite small subgroup of $\operatorname{GL}(2, k)$, which acts on $\mathbf{A}_{k}^{2}$ from the left. We assume throughout this article that the order $|G|$ of $G$ and the characteristic of $k$ are coprime.

Let $S=k[x, y]$ be the polynomial ring of two variables, and $R=S^{G}$ the subring of $S$ consisting of all $G$-invariants. Let $U:=\mathbf{A}_{k}^{2}=\operatorname{Spec} S$. $X=U / G=\operatorname{Spec} R$ and let $\pi: U \rightarrow X$ be the natural morphism. Since $G$ is small, the surface $X$ has a unique singular point 0 , which is a rational singularity [28]. Let $f: Y \rightarrow X$ be the minimal resolution of $X=U / G$. Thus we have a commutative diagram:

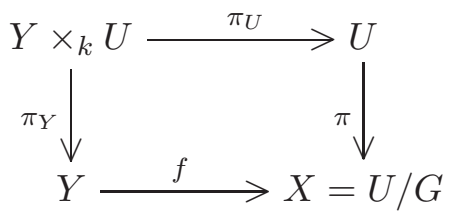

Let $F$ be the fundamental divisor of the singularity $(X, 0)$. That is, the minimum of effective divisors $D$ of $Y$ such that $D \neq 0, \operatorname{Supp}(D) \subset f^{-1}(0)$, and $D E^{\prime} \leq 0$ for any irreducible component $E^{\prime}$ of $f^{-1}(0)$.

The following is due to [33].

Theorem 2.8. Let $G$ be a finite small subgroup of $\mathrm{GL}(2, k)$ and $X=U / G$. Under the same notation as in Subsection 2.3,

(1) there is a bijective correspondence between the following sets

(i) the set of irreducible components $E_{i}$ of $E:=f^{-1}(0)$,

(ii) the set of non-trivial indecomposable full $\mathrm{O}_{Y}$-modules $\mathcal{M}_{i}$, special in the sense that $H^{1}\left(Y, \mathcal{M}_{i}^{\vee}\right)=0$,

where the correspondence $\mathcal{M}_{i} \mapsto E_{i}$ is given by

$$
c_{1}\left(\mathcal{M}_{i}\right) E_{j}=\delta_{i j},
$$

(2) the rank of $\mathcal{M}_{i}$ is equal to $c_{1}\left(\mathcal{M}_{i}\right) F$, the multiplicity of $E_{i}$ in $F$.

2.4. $G$-equivariant locally free $O_{U}$-modules and reflexive $O_{X}$-modules. Let $G$ be a small subgroup of $\mathrm{GL}(2, k)$. Any $G$-equivariant locally free $O_{U^{-}}$ module $\mathcal{F}$ of finite rank is of the form $\mathcal{F}=\widetilde{M}$ for a projective finite $S$-module $M$ with $G$-action. Note that $M$ is a free $S$-module ${ }^{1}$ by [26] and [27].

Let $M^{G}$ be the submodule consisting of all $G$-invariants of $M$. Then the natural exact sequence $0 \rightarrow M^{G} \rightarrow M$ of $R$-modules splits because the characteristic of $k$ is prime to the order $|G|$. In other words, there is an $R$-submodule $N$ of $M$ such that $M \simeq M^{G} \oplus N$.

\footnotetext{
${ }^{1} M$ is a projective $S$-module if and only if $M$ is a free $S$-module.
} 
For a $G$-equivariant locally free $O_{U}$-module $\mathcal{F}$, we define a subsheaf $\mathcal{F}^{G}$ of $\pi_{*}(\mathcal{F})$ consisting of $G$-invariant sections by

$$
\mathcal{F}^{G}(W)=\Gamma\left(\pi^{-1}(W), \mathcal{F}\right)^{G}=\Gamma\left(W, \pi_{*}(\mathcal{F})\right)^{G}
$$

for any open subscheme $W$ of $X$. Since $\pi$ is finite, $\mathcal{F}^{G}$ is a coherent $O_{X^{-}}$ submodule of $\pi_{*} \mathcal{F}$ associated to the $R$-module $M^{G}$. See $[6, \S 4]$ for more about $G$-sheaves. Let $U^{\prime}=U \backslash\{0\}, X^{\prime}=X \backslash\{\pi(0)\}, i: U^{\prime} \hookrightarrow U$ and $j: X^{\prime} \hookrightarrow X$ the natural inclusions.

Remark 2.9. If $\mathcal{F}$ is a $G$-equivariant locally free $O_{U}$-module, ${ }^{2} \mathcal{F}^{G}$ is a reflexive $O_{X^{-}}$-module. Conversely if $\mathcal{F}=\left(\pi^{*}(\mathcal{G})\right)^{\vee \vee}$ for a reflexive $O_{X}$-module $\mathcal{G}$, then $\mathcal{F}$ is a $G$-equivariant locally free $O_{U}$-module such that $\mathcal{F}^{G} \simeq \mathcal{G}$.

Definition 2.10. For a normal surface $Z$ over $k$, we denote by $\operatorname{Coh}(Z)$ (resp. $\mathrm{LF}(Z), \operatorname{Rfl}(Z)$ ) the category of coherent $O_{Z}$-modules, (resp. the category of locally free $O_{Z}$-modules of finite rank, the category of coherent reflexive $O_{Z^{-}}$ modules). If $Z$ has a $G$-action, then we denote by $\operatorname{Coh}^{G}(Z)\left(\operatorname{resp} \cdot \operatorname{LF}^{G}(Z)\right.$,

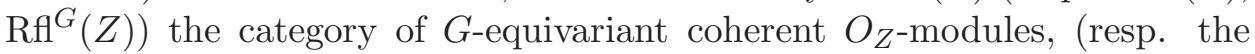
category of $G$-equivariant locally free $O_{Z}$-modules of finite rank, the category of $G$-equivariant coherent reflexive $O_{Z}$-modules). Let $G$ - $\operatorname{Hom}_{O_{Z}}(A, B)$ (resp. $\operatorname{Hom}_{O_{Z}}(A, B)$ ) be the set of all $G$-homomorphisms (resp. all homomorphisms) from $A$ to $B$ for $A, B \in \operatorname{Coh}^{G}(Z)$ (resp. $A, B \in \operatorname{Coh}(Z)$ ).

Lemma 2.11. The functor $G$-inv $: \mathcal{F} \mapsto \mathcal{F}^{G}$ is an equivalence of the categories $\operatorname{LF}^{G}(U)$ and $\operatorname{Rfl}(X)$.

Proof. Since $\pi^{\prime}: U^{\prime} \rightarrow X^{\prime}$ is flat surjective, by faithfully flat descent [11, Exposé VIII, Theorem 1.1], $\mathrm{Coh}^{G}\left(U^{\prime}\right)$ and $\operatorname{Coh}\left(X^{\prime}\right)$ are equivalent by the functor

$$
\mathcal{F} \mapsto \text { the descent of } \mathcal{F} \text { to } X^{\prime} \text {. }
$$

If $\mathcal{F}=\left(\pi^{\prime}\right)^{*} \mathcal{H}$ for a coherent $O_{X^{\prime}}$-module $\mathcal{H}$, then $\mathcal{F}^{G}=\left(\pi^{*} \mathcal{H}\right)^{G}=\mathcal{H}$. In other words, $\mathcal{F}^{G}$ is the descent of $\mathcal{F}$ to $X^{\prime}$, that is, $\mathcal{F}=\left(\pi^{\prime}\right)^{*}\left(\mathcal{F}^{G}\right)$. Moreover the category equivalence of $\operatorname{Coh}^{G}\left(U^{\prime}\right)$ and $\operatorname{Coh}\left(X^{\prime}\right)$ implies

$$
G-\operatorname{Hom}_{O_{U^{\prime}}}(\mathcal{F}, \mathcal{G})=\operatorname{Hom}_{O_{X^{\prime}}}\left(\mathcal{F}^{G}, \mathcal{G}^{G}\right) .
$$

By Remark 2.9, we see that $G$-inv $: \mathcal{F} \mapsto \mathcal{F}^{G}$ is an equivalence between $\mathrm{LF}^{G}\left(U^{\prime}\right)$ and $\operatorname{LF}\left(X^{\prime}\right)$. By Lemma $2.2(2), \operatorname{LF}\left(X^{\prime}\right)=\operatorname{Rf}\left(X^{\prime}\right)$. By Lemma 2.2 (2)-(4), the open immersion $i: U^{\prime} \hookrightarrow U$ induces an equivalence between $\mathrm{LF}^{G}\left(U^{\prime}\right)$ and $\mathrm{LF}^{G}(U)$. Similarly $j: X^{\prime} \hookrightarrow X$ induces an equivalence between $\operatorname{Rfl}\left(X^{\prime}\right)$ and $\operatorname{Rfl}(X)$.

Summarizing all the above, we see that $G$-inv $: \mathcal{F} \mapsto \mathcal{F}^{G}$ is an equivalence between $\operatorname{LF}^{G}(U)$ and $\operatorname{Rfl}(X)$. See also [3, Proposition 2.2].

\footnotetext{
${ }^{2} \mathcal{F}$ is a free $O_{U}$-module by [26] and [27].
} 
Definition 2.12. For an irreducible representation $\rho$ of $G$, we define

$$
\begin{aligned}
& M_{\rho}:=\left(O_{U} \otimes_{k} \rho^{*}\right)^{G}:=\left[\pi_{*}\left(O_{U}\right) \otimes_{k} \rho^{*}\right]^{G}, \\
& \mathcal{M}_{\rho}:=f^{*} M / O_{Y} \text {-torsions. }
\end{aligned}
$$

Corollary 2.13. Let $\rho, \sigma$ be irreducible representations of $G$, and $\rho^{*}$ the dual representation of $\rho$, Then $M_{\rho}$ is a reflexive $O_{X}$-module and

$$
\mathcal{H o m}_{O_{X}}\left(M_{\rho}, M_{\sigma}\right) \simeq M_{\rho^{*} \otimes_{k} \sigma} .
$$

Especially, the dual $O_{X}$-module $M_{\rho}^{\vee}$ of $M_{\rho}$ is isomorphic to $M_{\rho^{*}}$.

Definition 2.14. An irreducible representation $\rho$ of $G$ is said to be special if its corresponding full $O_{Y}$-module $\mathcal{M}:=\mathcal{M}_{\rho}:=\left[p_{*} q^{*}\left(O_{U} \otimes_{k} \rho^{*}\right)\right]^{G}$ is special, that is, $H^{1}\left(Y, \mathcal{M}^{\vee}\right)=0$. A $k[G]$-module $N$ is said to be special if $N$ is an irreducible $k[G]$-module isomorphic to a special representation.

We show the existence of non-special representations when $G \not \subset \mathrm{SL}(2, k)$.

Lemma 2.15. For a full sheaf $\mathcal{M}$ on $Y, \mathcal{M}$ is special if and only if $\mathcal{M} \otimes_{O_{Y}}$ $K_{Y}$ is a full sheaf.

Proof. Since $\mathcal{M}$ and $K_{Y}$ are generated by global sections by Corollary 2.7, so is $\mathcal{M} \otimes_{O_{Y}} K_{Y}$. Lemma follows from $H^{1}\left(\left(\mathcal{M} \otimes_{O_{Y}} K_{Y}\right)^{\vee} \otimes K_{Y}\right)=H^{1}\left(\mathcal{M}^{\vee}\right)$.

Lemma 2.16. If $G$ is not contained in $\mathrm{SL}(2, k)$, then the following is true.

(1) If $\mathcal{M}$ is an indecomposable special full sheaf such that $\mathcal{M} \otimes_{O_{Y}} K_{Y}$ is special, then $\mathcal{M} \simeq O_{Y}, K_{Y}$ is special, $K_{Y} F=1$ and $F^{2}=-3$.

(2) If $\rho \in \operatorname{Irr}(G)$ is special with $\rho \otimes_{k} \operatorname{det} \rho_{\text {nat }}^{\vee}$ special, then $\rho \simeq \rho_{0}$, $\operatorname{det} \rho_{\text {nat }}^{\vee}$ is special, $K_{Y} F=1$ and $F^{2}=-3$.

Proof. The assumption $G \not \subset \mathrm{SL}(2, k)$ implies that $K_{Y} F>0$, because otherwise, any irreducible component $E$ of $F$ is a smooth rational curve with $E^{2}=-2$, hence the singularity $U / G$ is one of $\mathrm{ADE}$, hence $G \subset \mathrm{SL}(2, k)$.

We shall prove (1). Assume that $\mathcal{M} \nsucceq O_{Y}$, and $\mathcal{M}$ is an indecomposable special full sheaf of rank $r$ such that $\mathcal{M} \otimes_{O_{Y}} K_{Y}$ is special. Then $r=c_{1}(\mathcal{M}) F$ and $r=c_{1}\left(\mathcal{M} \otimes_{O_{Y}} K_{Y}\right) F$ by Theorem 2.8 (2). This contradicts $K_{Y} F>0$. It follows that $\mathcal{M} \simeq O_{Y}$ and $K_{Y}$ is a special full sheaf of rank 1. Note that $K_{Y}$ is always a full sheaf by Corollary 2.7. We also note that $K_{Y}$ is special if and only if $K_{Y} F=1$ by Theorem 2.8 (2), which is equivalent to $F^{2}=-3$ because $H^{0}\left(O_{F}\right)=k$ and $H^{1}\left(O_{F}\right)=0$ by [1, Theorem 3]. For (2),

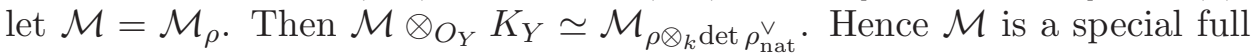
sheaf such that $\mathcal{M} \otimes_{O_{Y}} K_{Y}$ is special. Hence (2) follows from (1).

Corollary 2.17. Suppose $G$ is not contained in $\mathrm{SL}(2, k)$. Then for a nontrivial special representation $\rho, \rho \otimes \operatorname{det} \rho_{\text {nat }}^{\vee}$ is non-special. Especially, nonspecial representations exist. 
2.5. Universal $G$-cluster and full sheaves. The following is due to [19].

Theorem 2.18. Let $U=\mathbf{A}_{k}^{2}, G$ a finite subgroup of $\mathrm{GL}(2, k)$ such that the order $|G|$ of $G$ is prime to the characteristic of $k$. Then $Y=G-\operatorname{Hilb}(U)$ is connected and it is a minimal resolution of $X:=U / G$.

Definition 2.19. Let $\mathcal{Z}$ be the universal cluster over $Y=G$-Hilb $(U)$. Consider the commutative diagram:

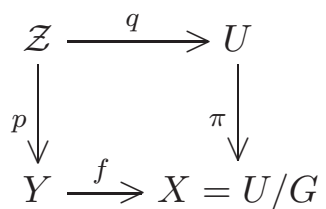

where $q:=\left(\pi_{U}\right)_{\mathcal{Z}}$ and $p:=\left(\pi_{Y}\right)_{\mathcal{Z}}$. Note that

$$
\mathcal{Z} \times_{Y}(Y \backslash F) \simeq\left(U \times_{X}(X \backslash\{O\}) .\right.
$$

Lemma 2.20. The following is true:

(1) $q_{*}\left(O_{\mathcal{Z}}\right)=O_{U}$ and $f_{*} p_{*}\left(O_{\mathcal{Z}}\right) \simeq \pi_{*} q_{*}\left(O_{\mathcal{Z}}\right)=\pi_{*}\left(O_{U}\right)$,

(2) $\pi_{*}\left(O_{U}\right)$ is a reflexive $O_{X}$-module,

(3) $p_{*}\left(O_{\mathcal{Z}}\right)$ is the torsion free pullback of $\pi_{*}\left(O_{U}\right)$ by $f$,

(4) for any irreducible representation $\rho$ of $G$,

$$
\mathcal{M}_{\rho} \cong\left[p_{*} q^{*}\left(O_{U}\right) \otimes_{k} \rho^{*}\right]^{G}=\left[p_{*}\left(O_{\mathcal{Z}}\right) \otimes_{k} \rho^{*}\right]^{G},
$$

(5) $p_{*}\left(O_{\mathcal{Z}}\right)=\bigoplus_{\rho \in \operatorname{Irr}(G)} \mathcal{M}_{\rho} \otimes_{k} \rho$ and $\pi_{*}\left(O_{U}\right)=\bigoplus_{\rho \in \operatorname{Irr}(G)} M_{\rho} \otimes_{k} \rho$.

Proof. Let $q^{\prime}: \mathcal{Z}^{\prime}:=\mathcal{Z} \backslash q^{-1}(0) \rightarrow U^{\prime}$ (resp. $p^{\prime}: \mathcal{Z}^{\prime} \rightarrow Y^{\prime}=Y \backslash F$ ) be the restriction of $q$ (resp. of $p$ ). First we prove $q_{*}\left(O_{\mathcal{Z}}\right)=O_{U}$. Since $q^{\prime}$ is an isomorphism, $\left(q^{\prime}\right)_{*}\left(O_{\mathcal{Z}^{\prime}}\right) \simeq O_{U^{\prime}}$. It follows that $O_{U} \subset q_{*}\left(O_{\mathcal{Z}}\right) \subset$ $i_{*}\left(\left(q^{\prime}\right)_{*}\left(O_{\mathcal{Z}^{\prime}}\right)\right)=i_{*}\left(O_{U^{\prime}}\right)=O_{U}$. Hence $q_{*}\left(O_{\mathcal{Z}}\right)=O_{U}$. This proves $(1)$.

Let $\mathcal{F}=\pi_{*}\left(O_{U}\right)$. Let $X^{\prime}=X \backslash\{0\}$ and $j: X^{\prime} \hookrightarrow X$. Then

$$
\Gamma\left(X, j_{*} \mathcal{F}\right)=\Gamma\left(X^{\prime}, \mathcal{F}\right)=\Gamma\left(U \backslash\{0\}, O_{U}\right)=\Gamma\left(U, O_{U}\right)=\Gamma(X, \mathcal{F}) .
$$

Since $X$ is affine, this shows $j_{*}(\mathcal{F})=\mathcal{F}$. By Lemma $2.2(4), \pi_{*}\left(O_{U}\right)=\mathcal{F}$ is reflexive. This proves (2).

Next we prove (3). See also the proof of [19, Theorem 3.1]. Since $\mathcal{Z}$ is finite and flat over $Y, p_{*}\left(O_{\mathcal{Z}}\right)$ is locally free. Moreover, it is generated by global sections because it is a quotient of the quasi-coherent $O_{Y}$-module $O_{Y} \otimes_{k} k[x, y]$. So it follows from $f_{*} p_{*}\left(O_{\mathcal{Z}}\right) \cong \pi_{*} O_{U}$ that $p_{*}\left(O_{\mathcal{Z}}\right)$ is the torsion free pullback of $\pi_{*}\left(O_{U}\right)$ by $f$.

Next we prove (4). It is proved in [19, Corollary 3.2] that $\left[p_{*}\left(O_{\mathcal{Z}}\right) \otimes_{k} \rho^{*}\right]^{G}$ is a full $O_{Y \text {-module. We have }}$

$$
\begin{aligned}
f_{*}\left(\left[p_{*}\left(O_{\mathcal{Z}}\right) \otimes_{k} \rho^{*}\right]^{G}\right) & =\left[f_{*} p_{*}\left(O_{\mathcal{Z}}\right) \otimes_{k} \rho^{*}\right]^{G}=\left[\pi_{*} q_{*}\left(O_{\mathcal{Z}}\right) \otimes_{k} \rho^{*}\right]^{G} \\
& =\left[\pi_{*}\left(O_{U}\right) \otimes_{k} \rho^{*}\right]^{G}=M_{\rho} .
\end{aligned}
$$

Taking the torsion-free pullbacks of the both sides, we obtain (4). 
Next we prove (5). By [6, Subsection 4.2] and since the characteristic of $k$ is prime to $|G|$, there is a decomposition $p_{*}\left(O_{\mathcal{Z}}\right)=\bigoplus_{\rho \in \operatorname{Irr}(G)} \mathcal{N}_{\rho} \otimes_{k} \rho$ for some $O_{Y}$-module $\mathcal{N}_{\rho}$. For any irreducible representation $\gamma$ of $G$, we have

$$
\mathcal{M}_{\gamma}:=\left[p_{*}\left(O_{\mathcal{Z}}\right) \otimes_{k} \gamma^{*}\right]^{G} \simeq \bigoplus_{\rho \in \operatorname{Irr}(G)} \mathcal{N}_{\rho} \otimes_{k}\left(\operatorname{Hom}_{k}(\gamma, \rho)\right)^{G} \simeq \mathcal{N}_{\gamma}
$$

The rest of (5) follows from (1) and (4).

\section{Global McKay Correspondence}

The purpose of this section is to prove Theorem 3.13 together with Corollary 3.14, which reformulate Theorem 1.1 in a more precise way. This is a global version of Theorem 3.6 proved in [19] in the sense that it describes a family of $k[G]$-modules in Theorem 3.6 parameterized by the exceptional set.

3.1. Local McKay correspondence. We first recall results from [19].

Definition 3.1. We define two functors

$$
\Psi: D^{G}(U) \rightarrow D(Y), \quad \Phi: D(Y) \rightarrow D^{G}(U)
$$

by

$$
\begin{aligned}
& \Psi(A)=\left[p_{*} \mathbf{L} q^{*}(A)\right]^{G}=\left[\mathbf{R}\left(\pi_{Y}\right)_{*}\left(O_{\mathcal{Z}} \stackrel{\mathbf{L}}{\otimes_{O_{Y \times{ }_{k} U}}} \pi_{U}^{*}(A)\right]^{G},\right. \\
& \Phi(B)=\mathbf{R}\left(\pi_{U}\right)_{*}\left(O_{\mathcal{Z}}^{\vee} \stackrel{\mathbf{L}}{\otimes_{O_{Y \times{ }_{k}} U}} \pi_{Y}^{*}\left(B \otimes_{k} \rho_{0}\right){\stackrel{\mathbf{L}}{O_{Y \times{ }_{k}}}} \pi_{U}^{*} K_{U}[2]\right) .
\end{aligned}
$$

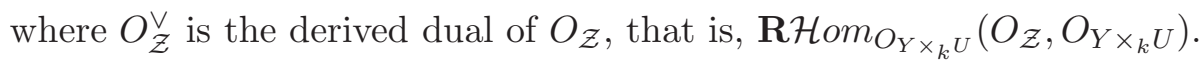

Theorem 3.2. $\Phi$ is fully faithful and $\Psi$ is a left adjoint of $\Phi$.

See $[19, \S 6]$ and $[20$, Proposition 1.1, Lemma 2.9].

Definition 3.3. We number all irreducible representations $\rho$ in the following manner: $\rho_{0}$ is trivial, $\rho_{i}(1 \leq i \leq m)$ is non-trivial special and $\rho_{j}(j \geq m+1)$ is non-trivial nonspecial.

We denote $\mathcal{M}_{\rho_{i}}$ by $\mathcal{M}_{i}$.

Lemma 3.4. Let $\rho_{i}$ be an irreducible special representation of $G, \mathcal{M}_{i}=$ $\mathcal{M}_{\rho_{i}}$, and $E_{i}$ the irreducible component of $F$ with $c_{1}\left(\mathcal{M}_{i}\right) E_{j}=\delta_{i j}$ as in Theorem 2.8. Then

$$
\Psi\left(O_{0} \otimes_{k} \rho^{*}\right)= \begin{cases}O_{E_{i}}(-1)[1] & \text { if } \rho=\rho_{i} \text { is non-trivial special } \\ O_{F} & \text { if } i=0, \text { that is, } \rho=\rho_{0} \text { is trivial, } \\ 0 & \text { if } \rho \text { is non-trivial, nonspecial. }\end{cases}
$$

See $[19, \S 5]$ for Lemma 3.4 . 
Lemma 3.5. Under the same notation as above,

$$
\begin{aligned}
& G \text { - } \operatorname{Ext}_{O_{U}}^{k}\left(O_{Z_{y}},\left(O_{0} \otimes_{k} \rho_{i}\right)\right):=\operatorname{Hom}_{D_{c}^{G}\left(O_{U}\right)}^{k}\left(O_{Z_{y}},\left(O_{0} \otimes_{k} \rho_{i}\right)\right) \\
& = \begin{cases}\operatorname{Ext}_{O_{Y}}^{k}\left(O_{F}, O_{y}\right) & (i=0) \\
\operatorname{Ext}_{O_{Y}}^{k-1}\left(O_{E_{i}}(-1), O_{y}\right) & (1 \leq i \leq m) \\
0 & (i \geq m+1)\end{cases} \\
& = \begin{cases}O_{y} & (i=0, k=0,1, y \in F), \\
O_{y} & \left(1 \leq i \leq m, k=1,2, y \in E_{i}\right) \\
0 & \text { (otherwise }) .\end{cases}
\end{aligned}
$$

See $[19, \S 7]$ for the above lemma. See also Definition 3.3.

Theorem 3.6. (Local McKay correspondence) Let $\mathfrak{m}$ be the maximal ideal of $O_{U}$ at the origin, $y \in Y$ and $Z_{y}$ the $G$-invariant cluster of $U$ corresponding to $y$ and $I_{Z_{y}}$ the ideal of $O_{U}$ defining $Z_{y}$. Then the $k[G]$-module $\operatorname{Gen}\left(I_{y}\right):=$ $I_{Z_{y}} / \mathfrak{m} I_{Z_{y}}$ is given by

$$
\begin{cases}\rho_{i} \oplus \rho_{0} & \text { if } y \in E_{i} \backslash \cup_{j \neq i} E_{j} \\ \rho_{i} \oplus \rho_{j} \oplus \rho_{0} & \text { if } y \in E_{i} \cap E_{j}, i \neq j .\end{cases}
$$

See [19, Theorem 7.1].

3.2. The ideals $n_{Y}$ and $I_{Y}$. Let $U=\mathbf{A}_{k}^{2}, X=U / G, Y=G$-Hilb $(U)$ and $f: Y \rightarrow X$ the natural morphism, which is the minimal resolution of $X$. Let $\pi_{Y}: Y \times_{k} U \rightarrow Y$ and $\pi_{U}: Y \times_{k} U \rightarrow U$ be the first and the second projection. Since $Y \times_{X} X \simeq Y$, we see that $Y$ is a closed subscheme of $Y \times_{k} X$. Let $I_{Y}$ be the ideal of $O_{Y \times_{k} X}$ defining $Y$, and $n_{Y}=I_{Y} O_{Y \times_{k} U}$.

Let $\mathcal{Z}$ be the universal subscheme of $Y \times_{k} U$, and $\mathcal{I}$ the ideal sheaf of $O_{Y \times{ }_{k} U}$ defining $\mathcal{Z}$. We have a commutative diagram of structure sheaves:

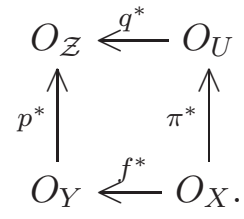

where

$$
\begin{aligned}
& O_{Y} \simeq O_{Y} \otimes_{O_{X}} O_{X} \simeq O_{Y} \otimes_{k} O_{X} / I_{Y}, \\
& O_{Y} \otimes_{k} O_{X} \stackrel{\mathrm{id}_{Y}^{*}}{\rightarrow} \pi^{*} O_{Y} \otimes_{k} O_{U} \stackrel{p^{*} \otimes q^{*}}{\rightarrow} O_{\mathcal{Z}}, \\
& O_{Y} \otimes_{k} O_{X} / I_{Y} \simeq O_{Y} \stackrel{p^{*}}{\rightarrow} O_{\mathcal{Z}}=O_{Y} \otimes_{k} O_{U} / \mathcal{I} .
\end{aligned}
$$

3.3. The fundamental divisor $F$. Let $F$ be the fundamental divisor of the singularity $(X, 0)$ (see Subsection 2.3).

Lemma 3.7. Let $\mathfrak{m}$ be the (maximal) ideal of $O_{U}$ defining the origin 0 , and $I_{F}$ the ideal of $O_{Y}$ defining the fundamental divisor $F$. Then 
(1) $O_{Y \times_{X} U} \simeq O_{Y} \otimes_{k} O_{U} / \mathfrak{n}_{Y}$, that is, $\mathfrak{n}_{Y}$ is the ideal of $O_{Y} \otimes_{k} O_{U}$ defining the fiber product $Y \times{ }_{X} U$.

(2) $\mathcal{Z} \subset Y \times_{X} U$, that is, $\mathfrak{n}_{Y} \subset \mathcal{I}$,

(3) $\pi_{U}^{*} \mathfrak{m}+\mathfrak{n}_{Y}=\pi_{U}^{*} \mathfrak{m}+I_{F} O_{Y \times_{k} U}=\pi_{U}^{*} \mathfrak{m}+\mathcal{I}$,

(4) $\mathfrak{m} \mathcal{I}+\mathfrak{n}_{Y} \mathcal{I}=\mathfrak{m} \mathcal{I}+\mathcal{I}^{2}=\mathfrak{m} \mathcal{I}+I_{F} \mathcal{I}$.

Proof. Let $\mathfrak{m}_{X}$ be the (maximal) ideal of $O_{X}$ defining the singular point 0 . The ideal $I_{Y}$ of $O_{Y} \otimes_{k} O_{X}$ is generated by $\left(f^{*} a\right) \otimes 1-1 \otimes a$ for $a \in \mathfrak{m}_{X}$. Therefore $\mathfrak{n}_{Y}$ is generated by $\left(f^{*} a\right) \otimes 1-1 \otimes\left(\pi^{*} a\right)$ for $a \in \mathfrak{m}_{X}$. Hence $O_{Y} \otimes_{k} O_{U} / \mathfrak{n}_{Y}$ is the structure sheaf of the fiber product $Y \times_{X} U$. Since $\mathcal{Z} \subset Y \times_{X} U$, we have $I_{Y} \subset \mathcal{I}$, so that $\mathfrak{n}_{Y} \subset \mathcal{I}$. This proves part (2).

The inclusion $\pi_{U}^{*} \mathfrak{m}+\mathfrak{n}_{Y} \subset \pi_{U}^{*} \mathfrak{m}+\mathcal{I}$ is clear. We shall prove the converse. Since $\mathfrak{n}_{Y}$ is generated by $\left(f^{*} a\right) \otimes 1-1 \otimes\left(\pi^{*} a\right)$ for $a \in \mathfrak{m}_{X}$, we have $\left(f^{*} a\right) \otimes 1 \in$ $\pi_{U}^{*} \mathfrak{m}+\mathfrak{n}_{Y}$ because $\pi^{*} a \in \mathfrak{m}$. Thus $\pi_{U}^{*} \mathfrak{m}+\mathfrak{n}_{Y}=\pi_{U}^{*} \mathfrak{m}+I_{F} O_{Y \times U}$. We see

$$
\begin{aligned}
O_{Y \times{ }_{k} U} /\left(\pi_{U}^{*} \mathfrak{m}+I_{F} O_{Y \times{ }_{k} U}\right) & \simeq\left(O_{Y} / I_{F}\right) \otimes_{O_{Y \times k_{k}}}\left(O_{U} / \mathfrak{m}\right)=O_{F} \\
O_{Y \times_{k} U} /\left(\pi_{U}^{*} \mathfrak{m}+\mathcal{I}\right) & \simeq\left(O_{Y \times_{k} U} / \mathcal{I}\right) \otimes_{O_{Y \times{ }_{k} U}}\left(O_{U} / \mathfrak{m}\right) \\
& \simeq O_{\mathcal{Z}} \otimes_{O_{Y \times k} U}\left(O_{U} / \mathfrak{m}\right)=O_{F}
\end{aligned}
$$

by Lemma $3.4(2)$. It follows that

$$
\pi_{U}^{*} \mathfrak{m}+I_{F} O_{Y \times U}=\pi_{U}^{*} \mathfrak{m}+\mathcal{I} .
$$

This proves Lemma.

Lemma 3.8. Let $\mathfrak{n}:=\left(\mathfrak{m} \cap k[x, y]^{G}\right) O_{U}$. Then the following is true:

(1) $I_{Z_{y}} \simeq \mathcal{I} / \mathfrak{m}_{y} \mathcal{I} \simeq\left(\mathcal{I} / \mathcal{I} \cap\left(\mathfrak{m}_{y} \otimes_{k} O_{U}\right)\right)$,

(2) $\mathcal{I} \cap\left(\mathfrak{m}_{y} \otimes_{k} O_{U}\right)=\mathfrak{m}_{y} \mathcal{I}$,

(3) $\mathfrak{n}_{Y}+\mathfrak{m}_{y} \otimes_{k} O_{U}=O_{Y} \otimes_{k} \mathfrak{n}+\mathfrak{m}_{y} \otimes_{k} O_{U}$ if $y \in F$,

(4) $\left(\mathfrak{n}_{Y}+\mathfrak{m}_{y} \mathcal{I}\right) / \mathfrak{m}_{y} \mathcal{I} \simeq \mathfrak{n}$ if $y \in F$.

Proof. Since $O_{\mathcal{Z}}$ is $O_{Y}$-flat,

$$
\begin{aligned}
O_{Z_{y}} & \simeq O_{\mathcal{Z}} \otimes_{O_{Y}} O_{y}=\left(O_{Y \times_{k} U} / \mathcal{I}\right) \otimes_{O_{Y}} O_{y} \\
& \simeq\left(O_{Y} \times_{k} O_{U}\right) /\left(\mathcal{I}+\mathfrak{m}_{y} \otimes_{k} O_{U}\right) \\
& \simeq\left(O_{y} \times_{k} O_{U}\right) /\left(\mathcal{I} / \mathcal{I} \cap\left(\mathfrak{m}_{y} \otimes_{k} O_{U}\right)\right),
\end{aligned}
$$

whence $I_{Z_{y}} \simeq \mathcal{I} / \mathcal{I} \cap\left(\mathfrak{m}_{y} \otimes_{k} O_{U}\right)$. This proves the part (1).

Since $O_{\mathcal{Z}}$ is $O_{Y}$-flat again, the following is an exact sequence:

$$
0 \rightarrow \mathcal{I} \otimes_{O_{Y \times_{k} U}} O_{y} \rightarrow O_{y} \otimes_{k} O_{U}\left(\simeq O_{U}\right) \rightarrow O_{Z_{y}}\left(\simeq O_{\mathcal{Z}} \otimes_{O_{Y \times_{k}}} O_{y}\right) \rightarrow 0,
$$

so that we have $I_{Z_{y}}=\mathcal{I} / \mathfrak{m}_{y} \mathcal{I}$. Hence we have

$$
\mathcal{I} \cap\left(\mathfrak{m}_{y} \otimes_{k} O_{U}\right)=\mathfrak{m}_{y} \mathcal{I},
$$

which proves the part (2). Since $\mathfrak{n}_{Y}$ is generated by $f^{*} a \otimes 1-1 \otimes \pi^{*} a$ for $a \in \mathfrak{m}_{X}$ and $\pi^{*} \mathfrak{m}_{X}=\mathfrak{n}$, we have $\mathfrak{n}_{Y}+\mathfrak{m}_{y} \otimes_{k} O_{U}=O_{Y} \otimes_{k} \mathfrak{n}+\mathfrak{m}_{y} \otimes_{k} O_{U}$ for 
$y \in F$, which is the part (3). It follows that

$$
\begin{aligned}
\left(\mathfrak{n}_{Y}+\mathfrak{m}_{y} \mathcal{I}\right) / \mathfrak{m}_{y} \mathcal{I} & \simeq \mathfrak{n}_{Y} / \mathfrak{n}_{Y} \cap \mathcal{I} \cap\left(\mathfrak{m}_{y} \otimes_{k} O_{U}\right) \\
& =\mathfrak{n}_{Y} / \mathfrak{n}_{Y} \cap\left(\mathfrak{m}_{y} \otimes_{k} O_{U}\right) \quad \text { by Lemma } 3.7(2) \\
& \simeq \mathfrak{n}_{Y}+\mathfrak{m}_{y} \otimes_{k} O_{U} /\left(\mathfrak{m}_{y} \otimes_{k} O_{U}\right) \\
& \simeq\left(O_{Y} \otimes_{k} \mathfrak{n}+\mathfrak{m}_{y} \otimes_{k} O_{U}\right) /\left(\mathfrak{m}_{y} \otimes_{k} O_{U}\right) \simeq \mathfrak{n} .
\end{aligned}
$$

This completes the proof.

Definition 3.9. We define

$$
\begin{aligned}
\mathcal{V} & :=\mathcal{I} /\left(\mathfrak{m} \mathcal{I}+\mathfrak{n}_{Y}\right), \\
\mathcal{V}^{\dagger} & :=\mathcal{I} /\left(\mathfrak{m} \mathcal{I}+I_{F} \mathcal{I}\right) \simeq(\mathcal{I} / \mathfrak{m} \mathcal{I}) \otimes_{O_{Y}} O_{F} .
\end{aligned}
$$

Lemma 3.10. For $y \in F$,

$$
\mathcal{V} \otimes_{O_{Y}} O_{y} \simeq I_{Z_{y}} /\left(\mathfrak{m} I_{Z_{y}}+\mathfrak{n}\right) ; \mathcal{V}^{\dagger} \otimes_{O_{Y}} O_{y} \simeq I_{Z_{y}} / \mathfrak{m} I_{Z_{y}}
$$

Proof. Let $y \in Y$ and $\mathfrak{m}_{y}$ the maximal ideal of $O_{Y, y}$. Hence $O_{y}=O_{Y, y} / \mathfrak{m}_{y}=$ $O_{y}$. Since $I_{Z_{y}}=\mathcal{I} / \mathfrak{m}_{y} \mathcal{I}$, we have

$$
\begin{aligned}
\mathcal{V} \otimes_{O_{Y}} O_{y} & \simeq\left(\mathcal{I} /\left(\mathfrak{m} \mathcal{I}+\mathfrak{n}_{Y}\right)\right) \otimes_{O_{Y}} O_{y}=\mathcal{I} /\left(\mathfrak{m} \mathcal{I}+\mathfrak{n}_{Y}+\mathfrak{m}_{y} \mathcal{I}\right) \\
& \left.\simeq\left(\mathcal{I} / \mathfrak{m}_{y} \mathcal{I}\right) /\left(\mathfrak{m}\left(\mathcal{I} / \mathfrak{m}_{y} \mathcal{I}\right)+\left(\mathfrak{n}_{Y}+\mathfrak{m}_{y} \mathcal{I}\right) / \mathfrak{m}_{y} \mathcal{I}\right)\right) \\
& \simeq I_{Z_{y}} /\left(\mathfrak{m} I_{Z_{y}}+\mathfrak{n}\right) \\
\mathcal{V}^{\dagger} \otimes_{O_{Y}} O_{y} & =\mathcal{I} /\left(\mathfrak{m} \mathcal{I}+I_{F} \mathcal{I}+\mathfrak{m}_{y} \mathcal{I}\right)=\mathcal{I} /\left(\mathfrak{m} \mathcal{I}+\mathfrak{m}_{y} \mathcal{I}\right) \\
& \left.\simeq\left(\mathcal{I} / \mathfrak{m}_{y} \mathcal{I}\right) /\left(\mathfrak{m} \mathcal{I}+\mathfrak{m}_{y} \mathcal{I}\right) / \mathfrak{m}_{y} \mathcal{I}\right) \\
& \simeq\left(\mathcal{I} / \mathfrak{m}_{y} \mathcal{I}\right) / m\left(\mathcal{I} / \mathfrak{m}_{y} \mathcal{I}\right) \simeq I_{Z_{y}} / \mathfrak{m} I_{Z_{y}} .
\end{aligned}
$$

Definition 3.11. For a coherent $O_{Y \times U}$-module $J$, we define a functor

$$
\Psi_{J}: D_{c}^{G}(U) \rightarrow D_{c}(Y)
$$

by

$$
\left.\Psi_{J}(A)=\left[p_{*}\left(\mathbf{L} \pi_{U}^{*}(A) \stackrel{\mathbf{L}}{\otimes_{O_{Y \times_{k}}}} J\right)\right]^{G}=\mathbf{R}\left(\pi_{Y}\right)_{*}\left(\mathbf{L} \pi_{U}^{*}(A) \stackrel{\mathbf{L}}{\otimes_{O_{Y \times_{k}}}} J\right)\right]^{G}
$$

where $A \in D_{c}^{G}(U)$. Note that $\Psi=\Psi_{O_{\mathcal{Z}}}$.

Lemma 3.12. The following is true:

(1) $\Psi\left(O_{0} \otimes_{k} \rho_{i}^{*}\right)= \begin{cases}O_{F} & (i=0), \\ O_{E_{i}}(-1)[1] & \left(\rho_{i}: \text { non-trivial special }\right)\end{cases}$

(2) $\Psi_{O_{Y \times_{k} U}}\left(O_{0} \otimes_{k} \rho_{i}^{*}\right)= \begin{cases}O_{Y} & (i=0), \\ 0 & (i \neq 0),\end{cases}$

(3) $\Psi_{\mathcal{I}}\left(O_{0} \otimes_{k} \rho_{i}^{*}\right)= \begin{cases}O_{Y}(-F) & (i=0) \\ O_{E_{i}}(-1) & (i \neq 0) \\ 0 & \text { (otherwise). }\end{cases}$ 
Proof. The part (1) follows from Lemma 3.4. Since $O_{Y \times_{k} U}$ is $O_{Y \times_{k} U}$-flat, by definition,

$$
\begin{aligned}
\Psi_{O_{Y \times_{k} U}}\left(O_{0} \otimes_{k} \rho_{i}^{*}\right) & =\left[p_{*}\left(O_{Y} \otimes_{k}\left(O_{0} \otimes_{k} \rho_{i}^{*}\right)\right)\right]^{G} \\
& =\left[O_{Y} \otimes_{k} \rho_{i}^{*}\right]^{G}= \begin{cases}O_{Y} & (i=0) \\
0 & (i \neq 0)\end{cases}
\end{aligned}
$$

The part (3) follows from the parts (1) and (2) and the exact sequence:

$$
\begin{aligned}
& \longrightarrow \Psi_{\mathcal{I}}^{-2}\left(O_{0} \otimes_{k} \rho_{0}^{*}\right) \rightarrow \Psi_{O_{Y \times_{k} U}}^{-2}\left(O_{0} \otimes_{k} \rho_{0}^{*}\right) \rightarrow \Psi_{O \mathcal{Z}}^{-2}\left(O_{0} \otimes_{k} \rho_{0}^{*}\right) \\
& \longrightarrow \Psi_{\mathcal{I}}^{-1}\left(O_{0} \otimes_{k} \rho_{0}^{*}\right) \rightarrow \Psi_{O_{Y \times_{k} U}}^{-1}\left(O_{0} \otimes_{k} \rho_{0}^{*}\right) \rightarrow \Psi_{O_{\mathcal{Z}}}^{-1}\left(O_{0} \otimes_{k} \rho_{0}^{*}\right) \\
& \longrightarrow \Psi_{\mathcal{I}}^{0}\left(O_{0} \otimes_{k} \rho_{0}^{*}\right) \rightarrow \Psi_{O_{Y \times_{k} U}}^{0}\left(O_{0} \otimes_{k} \rho_{0}^{*}\right) \rightarrow \Psi_{O_{\mathcal{Z}}}^{0}\left(O_{0} \otimes_{k} \rho_{0}^{*}\right) \longrightarrow 0 .
\end{aligned}
$$

This proves the part (3).

The following is a global version (global over the exceptional set) of Theorem 3.6:

Theorem 3.13. There are isomorphisms

$$
\mathcal{V} \simeq \sum_{\rho_{i} \neq \rho_{0}} O_{E_{i}}(-1) \otimes_{k} \rho_{i}, \quad \mathcal{V}^{\dagger} \simeq \mathcal{V} \oplus O_{F}(-F) \otimes_{k} \rho_{0},
$$

where $\rho_{i}$ ranges over all non-trivial special irreducible representations of $G$.

Corollary 3.14. Let $\mathfrak{n}=\left(\mathfrak{m} \cap k[x, y]^{G}\right) O_{U}$. Then the fibers of $\mathcal{V}$ and $\mathcal{V}^{\dagger}$ over $y \in F$ are

$$
\begin{aligned}
\mathcal{V} \otimes_{O_{Y}} O_{y} & = \begin{cases}\rho_{i} & \left(y \in E_{i} \backslash \cup_{j \neq i} E_{j}\right) \\
\rho_{i} \oplus \rho_{j} & \left(y \in E_{i} \cap E_{j}, i \neq j\right),\end{cases} \\
\mathcal{V}^{\dagger} \otimes_{O_{Y}} O_{y} & = \begin{cases}\rho_{i} \oplus \rho_{0} & \left(y \in E_{i} \backslash \cup_{j \neq i} E_{j}\right) \\
\rho_{i} \oplus \rho_{j} \oplus \rho_{0} & \left(y \in E_{i} \cap E_{j}, i \neq j\right) .\end{cases}
\end{aligned}
$$

We note $E_{i}=C\left(\rho_{i}\right)$ and $\operatorname{Gen}\left(I_{Z_{y}}\right)=I_{Z_{y}} / \mathfrak{m} I_{Z_{y}}$ in Theorem 1.1.

Proof of Theorem 3.13 and Corollary 3.14. By Lemma 3.12, we have

$$
\begin{aligned}
\mathcal{I} / \mathfrak{m} \mathcal{I} & =p_{*}\left(\mathbf{L} \pi_{U}^{*}\left(O_{0}\right){\stackrel{\mathbf{L}}{O_{Y \times_{k} U}}} \mathcal{I}\right) \simeq \sum_{\rho: \text { irred. }} \Psi_{\mathcal{I}}\left(O_{0} \otimes_{k} \rho^{*}\right) \otimes \rho \\
& \simeq \bigoplus_{i=1}^{n} O_{E_{i}}(-1) \otimes_{k} \rho_{i} \bigoplus O_{Y}(-F) \otimes_{k} \rho_{0},
\end{aligned}
$$

which is an isomorphism in $D_{c}(Y)$. However since the rhs is concentrated to degree zero only, it is an isomorphism of $O_{Y}$-modules. It follows

$$
\begin{aligned}
\mathcal{V}^{\dagger} & =\mathcal{I} /\left(\mathfrak{m} \mathcal{I}+I_{F} \mathcal{I}\right) \simeq(\mathcal{I} / \mathfrak{m} \mathcal{I}) \otimes_{O_{Y}} O_{F} \\
& \simeq \bigoplus_{i=1}^{n} O_{E_{i}}(-1) \otimes_{k} \rho_{i} \bigoplus O_{F}(-F) \otimes_{k} \rho_{0} .
\end{aligned}
$$


It remains to compute $\mathcal{V}$. By Lemma 3.10,

$$
\begin{aligned}
I_{Z_{y}} / \mathfrak{m} I_{Z_{y}} & =\bigoplus_{i=1}^{n} O_{E_{i}}(-1) \otimes_{O_{Y}} O_{y} \otimes_{k} \rho_{i} \bigoplus O_{y}(-F) \otimes_{k} \rho_{0}, \\
I_{Z_{y}} /\left(\mathfrak{m} I_{Z_{y}}+\mathfrak{n}\right) & \simeq \bigoplus_{i=1}^{n} O_{E_{i}}(-1) \otimes_{O_{Y}} O_{y} \otimes_{k} \rho_{i}
\end{aligned}
$$

where every generator of $O_{F}(-F) \otimes_{k} \rho_{0} \subset \mathcal{V}^{\dagger}$ is the image of a $G$-invariant polynomial in $\mathcal{I}$, which reduces to zero in $\mathcal{V}$ because $\mathfrak{m} \cap k[x, y]^{G} \subset \mathfrak{n}$. Hence we have $\mathcal{V}=\bigoplus_{i=1}^{n} O_{E_{i}}(-1) \otimes_{k} \rho_{i}$. This proves Theorem 3.13 and Corollary 3.14.

Remark 3.15. Whether or not one can find an isomorphism similar to Theorem 3.13 is of some interest when $U=\mathbf{C}^{n}$ and $G$ is a very natural subgroup of $\mathrm{GL}(U)$. For example, $U$ is the Griess algebra or the vertex operator algebra for the big Monster M. For $G=M_{24}$, the Mathieu group of degree $24, U$ is the Leech lattice.

3.4. Integral functors with dual kernel objects. In this subsection, we slightly alter the functors $\Phi$ and $\Psi$ into functors which are more suitable for our purpose.

For $J \in D^{G}(Y \times U)$, we define a functor $\Phi_{J}: D_{c}(Y) \rightarrow D_{c}^{G}(U)$ by

$$
\Phi_{J}(-)=\mathbf{R}\left(\pi_{U}\right)_{*}\left(\pi_{Y}^{*}(-) \stackrel{\mathbf{L}}{\left.\otimes_{O_{Y \times_{k}}} J\right) .}\right.
$$

In the sequel, we assume that the support of $J$ is proper over both $Y$ and $U$. Then $\Phi_{J}$ is extended to

$$
\Phi_{J}: D(Y) \rightarrow D^{G}(U) .
$$

We denote the right adjoint of $\Phi_{J}$ by $\Phi_{J}^{*}$.

Lemma 3.16. Let $J$ be an object of $D^{G}\left(Y \times_{k} U\right)$ whose support is proper over both $Y$ and $U, J^{\vee}$ the derived dual of $J, A \in D(Y)$ and $A^{\vee}$ the derived dual of $A$. Then

$$
\Phi_{J \vee}(A) \simeq \Phi_{J}\left(A^{\vee} \stackrel{\mathrm{L}}{\otimes_{O_{Y}}} K_{Y}[2]\right)^{\vee} .
$$

Proof. The assertion follows from:

$$
\begin{aligned}
\Phi_{J \vee}(A)^{\vee} & \simeq \mathbf{R} \operatorname{Hom}_{O_{U}}\left(\mathbf{R}\left(\pi_{U}\right)_{*}\left(\pi_{Y}^{*}(A) \stackrel{\mathrm{L}}{\otimes_{O_{Y \times_{k}}}} J^{\vee}\right), O_{U}\right) \\
& \simeq \mathbf{R}\left(\pi_{U}\right)_{*}\left(\mathbf{R} H \operatorname{Hom}_{O_{Y \times} U}\left(\pi_{Y}^{*}(A) \stackrel{\mathrm{L}}{\otimes_{O_{Y \times} U}} J^{\vee}, \pi_{Y}^{*} K_{Y}[2]\right)\right) \\
& \simeq \mathbf{R}\left(\pi_{U}\right)_{*}\left(\pi_{Y}^{*}\left(A^{\vee} \stackrel{\mathrm{L}}{\otimes}_{O_{Y}} K_{Y}[2]\right) \stackrel{\mathrm{L}}{\otimes}_{O_{Y \times_{k} U}} J\right) \\
& \simeq \Phi_{J}\left(A^{\vee} \stackrel{\mathrm{L}}{\otimes}_{O_{Y}} K_{Y}[2]\right) .
\end{aligned}
$$

See also [17, Remark 5.8]. 
Corollary 3.17. $\Phi_{O_{\mathcal{Z}}}(B) \simeq \Phi\left(B^{\vee} \otimes_{O_{Y}} K_{Y}\right)^{\vee} \otimes_{O_{Y \times{ }_{k} U}} \pi_{U}^{*} K_{U}$.

Corollary 3.18. Let $\phi$ and $\psi$ be the same as in Definition 3.1. Then the right adjoint $\Phi_{O_{\mathcal{Z}}}^{*}$ of $\Phi_{O_{\mathcal{Z}}}$ is given by

$$
\Phi_{O_{\mathcal{Z}}}^{*}(B)=\Psi\left(B^{\vee} \stackrel{\mathrm{L}}{\otimes}_{O_{U}} K_{U}\right)^{\vee} \stackrel{\mathrm{L}}{\otimes_{O_{Y}}} K_{Y}
$$

for $A \in D(Y)$ and $B \in D_{c}^{G}(U)$.

Proof. The assertion follows from:

$$
\begin{aligned}
& \operatorname{Hom}_{D^{G}(U)}\left(\Phi_{O_{\mathcal{Z}}}(A), B\right) \simeq \operatorname{Hom}_{D^{G}(U)}\left(B^{\vee}, \Phi_{O_{\mathcal{Z}}}(A)^{\vee}\right) \\
& \simeq \operatorname{Hom}_{D^{G}(U)}\left(B^{\vee} \stackrel{\mathrm{L}}{\otimes} O_{U} K_{U}, \Phi_{O_{\mathcal{Z}}}(A)^{\vee} \stackrel{\mathrm{L}}{\otimes_{O_{U}}} K_{U}\right) \\
& \simeq \operatorname{Hom}_{D^{G}(U)}\left(B^{\vee} \stackrel{\mathbf{L}}{\otimes} O_{U} K_{U}, \Phi\left(A^{\vee} \stackrel{\mathrm{L}}{\otimes} O_{Y} K_{Y}\right)\right) \quad(\because \text { by Corollary } 3.17) \\
& \simeq \operatorname{Hom}_{D(Y)}\left(\Psi\left(B^{\vee} \stackrel{\mathbf{L}}{\otimes} O_{U} K_{U}\right), A^{\vee} \stackrel{\mathbf{L}}{\otimes_{O_{Y}}} K_{Y}\right) \quad(\because \text { by Theorem } 3.2) \\
& \simeq \operatorname{Hom}_{D(Y)}\left(A, \Psi\left(B^{\vee} \stackrel{\mathbf{L}}{\otimes_{O_{U}}} K_{U}\right)^{\vee} \stackrel{\mathbf{L}}{\otimes_{O_{Y}}} K_{Y}\right) \text {. }
\end{aligned}
$$

Now Lemma 3.4 (see also [19, Theorem 5.1]) is restated as follows:

Corollary 3.19. For an irreducible representation $\rho$ of $G$,

$$
\Phi_{O_{\mathcal{Z}}}^{*}\left(O_{0} \otimes_{k} \rho\right) \simeq \begin{cases}O_{E(\rho)}(-1) & \text { if } \rho \text { is non-trivial special, } \\ \omega_{F}[1] & \text { if } \rho=\rho_{0}, \\ 0 & \text { otherwise. }\end{cases}
$$

Here, for a special representation $\rho=\rho_{i}, E(\rho)=E_{i}$ denotes the corresponding irreducible component of $F$.

\section{Extensions of the Socle And the Cup PRoducts}

\subsection{Basic construction of extensions.}

Definition 4.1. Let

$$
\text { (s) } \quad: \quad 0 \longrightarrow A \stackrel{\phi}{\longrightarrow} C \stackrel{\eta}{\longrightarrow} F \longrightarrow 0 .
$$

be an exact sequence of $R$-modules. For any $R$-homomorphism $\psi: A \rightarrow B$, we define the pushforward $\psi_{*}(s)$ of $(s)$ by $\psi$ to be an exact sequence:

$$
\psi_{*}(s): \quad 0 \longrightarrow B \stackrel{\left(\mathrm{id}_{B}, 0\right)}{\longrightarrow} C_{\psi} \stackrel{\eta \cdot p_{2}}{\longrightarrow} F \longrightarrow 0 .
$$

where $C_{\psi}:=B \times C /(\psi,-\phi)(A)$.

Definition 4.2. For any $R$-homomorphism $\gamma: E \rightarrow F$, we define the pullback $\gamma^{*}(s)$ of $(s)$ by $\gamma$ to be an exact sequence:

$$
\gamma^{*}(s): \quad 0 \longrightarrow A \stackrel{(0, \phi)}{\longrightarrow} C^{\gamma} \stackrel{\eta \cdot p_{2}}{\longrightarrow} E \longrightarrow 0
$$

where $C^{\gamma}:=E \times{ }_{F} C:=\{(e, c) \in E \times C ; \gamma(e)=\eta(c)\}$. 
Definition 4.3. For an $O_{U, 0}$-module $M$, we define the socle $\operatorname{Soc}(M)$ of $M$ to be the sum of all minimal $O_{U, 0}$-submodules of $M$. For a cluster $Z_{y}$ with $y \in F, O_{Z_{y}}=O_{U} / I_{y}$ is an $O_{U}$-module supported by the origin of $U$. Let $\mathfrak{m}$ be the maximal ideal of $O_{U}$ defining $O$. Then it is easy to see

$$
\operatorname{Soc}\left(O_{Z_{y}}\right)=\left[I_{y}: \mathfrak{m}\right] / I_{y} .
$$

4.2. The extension $O_{U} / \mathfrak{m} I_{y}$. The purpose of this subsection is to study the following exact sequence:

$$
\begin{gathered}
\left(t_{1}\right): \quad 0 \longrightarrow I_{y} / \mathfrak{m} I_{y} \longrightarrow O_{U} / \mathfrak{m} I_{y} \stackrel{\phi}{\longrightarrow} O_{Z_{y}} \longrightarrow 0 \\
\| \quad \cup \quad 0 \\
\left(t_{2}\right): \quad 0 \longrightarrow I_{y} / \mathfrak{m} I_{y} \rightarrow\left[I_{y}: \mathfrak{m}\right] / \mathfrak{m} I_{y} \rightarrow\left[I_{y}: \mathfrak{m}\right] / I_{y} \longrightarrow 0 .
\end{gathered}
$$

Let $\operatorname{Gen}\left(I_{y}\right)=I_{y} / \mathfrak{m} I_{y}=\bigoplus_{\rho \in \Lambda_{y}} W(\rho)$ for some $G$-invariant $G$-irreducible $O_{U}$-submodule $W(\rho) \neq 0$ where $\Lambda_{y}$ is the set of irreducible representations appearing as direct summands in Theorem 3.6.

By Lemma 3.5, for every $\rho \in \Lambda_{y}$, there exists a unique ideal $J_{\rho}$ of $O_{U}$ with $\mathfrak{m} I_{y} \subset J_{\rho} \subset I_{y}$ and a non-trivial extension

$$
\left(\operatorname{ext}_{\rho}\right): \quad 0 \longrightarrow W(\rho) \rightarrow O_{U} / J_{\rho} \rightarrow O_{Z_{y}} \longrightarrow 0
$$

where $I_{y} / J_{\rho} \simeq W(\rho)$ and $\mathfrak{m} I_{y}=\cap_{\rho \in \Lambda_{y}} J_{\rho}$ because $\operatorname{Gen}\left(I_{y}\right)=\bigoplus_{\rho \in \Lambda_{y}} W(\rho)$.

We choose and fix $0 \neq V(\xi) \subset \operatorname{Soc}\left(O_{Z_{y}}\right)$ with $V(\xi) \cong \xi$. Since $V(\xi)$ is an $O_{U}$-submodule of $O_{Z_{y}}$, there exists an $O_{U}$-submodule $B(\xi)$ of $\left[I_{y}: \mathfrak{m}\right]$ such that $B(\xi) / I_{y}=V(\xi)$. Then we have a commutative diagram of exact sequences

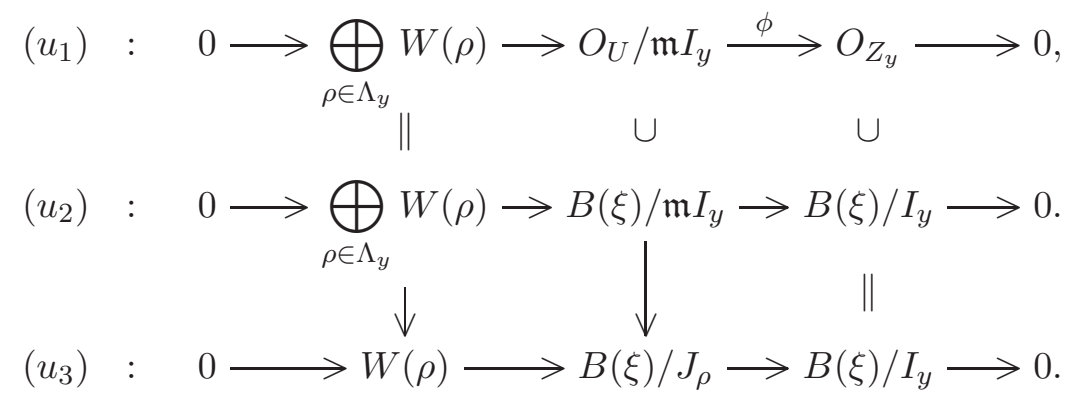

It is easy to see

Lemma 4.4. Let $V_{\rho}^{\natural}(\xi)$ be any $k[G]$-submodule (not necessarily an $O_{U^{-}}$ submodule) of $B(\xi) / J_{\rho}$ lifting $V(\xi)$. Then the following are equivalent:

(1) the exact sequence $\left(u_{3}\right)$ does not split,

(2) no lifting $V_{\rho}^{\natural}(\xi)$ of $V(\xi)$ is a $G$-invariant $O_{U}$-module,

(3) $S_{1} \cdot V_{\rho}^{\natural}(\xi)=W(\rho)$,

(4) $S_{1} \cdot V_{\rho}^{\natural}(\xi) \supset W(\rho)$. 
4.3. Cup-products. We choose and fix irreducible representations $\rho$ and $\xi$ of $G$ such that $W(\rho) \subset \operatorname{Gen}\left(I_{y}\right)$ and $V(\xi) \subset \operatorname{Soc}\left(O_{Z_{y}}\right)$.

The extension $\left(\operatorname{ext}_{\rho}\right)$ is uniquely determined by $\rho$ in view of Theorem 3.6. Since every irreducible submodule $V(\xi)$ of $O_{Z_{y}}$ is unique, the pullback $\left(u_{3}\right)$ of $\left(\operatorname{ext}_{\rho}\right)$ via the inclusion $j: V(\xi) \hookrightarrow O_{Z_{y}}$ is written as:

$$
0 \longrightarrow W(\rho) \rightarrow B(\xi) / J_{\rho} \rightarrow V(\xi)=B(\xi) / I_{y} \longrightarrow 0 .
$$

Associated to (4.3.1), we have a natural cup product

$$
\begin{aligned}
\operatorname{Hom}_{D^{G}(U)}^{0}\left(O_{0} \otimes_{k} \xi, O_{Z_{y}}\right) & \times \operatorname{Hom}_{D^{G}(U)}^{1}\left(O_{Z_{y}}, O_{0} \otimes_{k} \rho\right) \\
& \rightarrow \operatorname{Hom}_{D^{G}(U)}^{1}\left(O_{0} \otimes_{k} \xi, O_{0} \otimes_{k} \rho\right) .
\end{aligned}
$$

By Lemma 4.4, the following are equivalent:

(d) the cup product (4.3.2) is nonzero,

(e) the extension (4.3.1) is non-trivial,

(f) $S_{1} \cdot V_{\rho}^{\natural}(\xi) \supset W(\rho)$.

4.4. The case where $G \subset \mathrm{SL}(2)$. In this subsection, we assume $G \subset \mathrm{SL}(2)$. Since $G \subset \operatorname{SL}(2)$, both $\Psi$ and $\Phi$ are equivalences of the categories such that $\Psi \Phi \cong \operatorname{id}_{D(Y)}$ and $\Phi \Psi \cong \operatorname{id}_{D^{G}(U)}$. This is proved by the same argument as in [6] and [19, Theorem 6.2]. The functors $\Phi_{O_{\mathcal{Z}}}$ and its adjoint $\Phi_{O_{\mathcal{Z}}}^{*}$ are also equivalences by Corollary 3.17 and 3.18 (actually, [6] considers $\Phi_{O_{\mathcal{Z}}}$ and $\Phi_{O z}^{*}$ rather than $\Phi$ and $\Psi$ in this paper).

In what follows we consider a pair $\xi$ and $\rho$ in $\operatorname{Irr}(G)$ such that $\xi \subset$ $\operatorname{Soc}\left(O_{Z_{y}}\right)$ and $\rho \subset \operatorname{Gen}\left(I_{y}\right)$. Since $G \subset \operatorname{SL}(2), \xi$ is special and non-trivial.

We consider the cup product (4.3.2) in $D^{G}(U)$

$$
\begin{aligned}
\operatorname{Hom}_{D^{G}(U)}^{0}\left(O_{0} \otimes_{k} \xi, O_{Z_{y}}\right) & \times \operatorname{Hom}_{D^{G}(U)}^{1}\left(O_{Z_{y}}, O_{0} \otimes_{k} \rho\right) \\
& \rightarrow \operatorname{Hom}_{D^{G}(U)}^{1}\left(O_{0} \otimes_{k} \xi, O_{0} \otimes_{k} \rho\right) .
\end{aligned}
$$

If $\rho \neq \rho_{0}, \xi \neq \rho_{0}$, then it is translated by Corollary 3.19 into the following cup product

$$
\begin{aligned}
\operatorname{Hom}_{O_{Y}}\left(O_{E(\rho)}(-1),\right. & \left.O_{y}\right) \times \operatorname{Ext}_{O_{Y}}^{1}\left(O_{y}, O_{E(\xi)}(-1)\right) \\
& \rightarrow \operatorname{Ext}_{O_{Y}}^{1}\left(O_{E(\rho)}(-1), O_{E(\xi)}(-1)\right) .
\end{aligned}
$$

If $\rho=\rho_{0}$ and $\xi \neq \rho_{0}$, then (4.3.2) is translated into

$$
\begin{aligned}
\operatorname{Hom}_{O_{Y}}\left(O_{E(\xi)}(-1),\right. & \left.O_{y}\right) \times \operatorname{Ext}_{O_{Y}}^{2}\left(O_{y}, \omega_{F}\right) \\
& \rightarrow \operatorname{Ext}_{O_{Y}}^{2}\left(O_{E(\xi)}(-1), \omega_{F}\right)
\end{aligned}
$$

and then, by Serre duality, into the following:

$$
\begin{aligned}
\operatorname{Hom}_{O_{Y}}\left(\omega_{F}, O_{E(\xi)}\right. & (-1)) \times \operatorname{Hom}_{O_{Y}}\left(O_{E(\xi)}(-1), O_{y}\right) \\
& \rightarrow \operatorname{Hom}_{O_{Y}}\left(\omega_{F}, O_{y}\right) .
\end{aligned}
$$

In order to calculate these cup products, we use the following lemmas which holds for the minimal resolutions of rational surface singularities: 
Lemma 4.5. Suppose $G \subset \mathrm{GL}(2)$. Let $F$ be the fundamental divisor, and $C$ any irreducible component of $F$. Then we have

$$
\operatorname{Ext}_{O_{Y}}^{q}\left(O_{y}, O_{C}(-1)\right) \simeq \operatorname{Ext}_{O_{Y}}^{2-q}\left(O_{C}(-1), O_{y}\right)^{\vee}= \begin{cases}k & (q=1,2, y \in C) \\ 0 & (\text { otherwise })\end{cases}
$$

Lemma 4.6. Suppose $G \subset \mathrm{GL}(2)$. Let $F$ be the fundamental divisor, and $E, C$ any irreducible component of $F$ with $E \neq C$. Then we have

$$
\begin{aligned}
\operatorname{Ext}_{O_{Y}}^{q}\left(O_{C}(-1), O_{E}(-1)\right) & = \begin{cases}k & (q=1, C E=1) \\
0 & (\text { otherwise })\end{cases} \\
\operatorname{Ext}_{O_{Y}}^{q}\left(O_{E}(-1), O_{E}(-1)\right) & = \begin{cases}k & (q=0) \\
k^{\oplus 1} & \left(q=2, E^{2}=-2-d\right) \\
0 & (\text { otherwise })\end{cases} \\
\operatorname{Ext}_{O_{Y}}^{q}\left(O_{F}, O_{E}(-1)\right) & = \begin{cases}k^{\oplus e} & (q=2, F E=-e<0) \\
0 & (q=2, F E=0) \\
0 & (\text { otherwise })\end{cases}
\end{aligned}
$$

where $d=0$ and $e \leq 2$ if $G \subset \mathrm{SL}(2)^{3}$.

The proofs of the lemmas are easy, so we omit them.

Lemma 4.7. Suppose $G \subset \mathrm{SL}(2)$. Then the following is true:

(1) the cup product of (4.4.1) is $\begin{cases}\neq 0 & \text { if }\{y\}=E(\xi) \cap E(\rho) \\ 0 & \text { (otherwise) }\end{cases}$

(2) the cup product of (4.4.2) is $\begin{cases}\neq 0 & \text { if } F E(\xi) \neq 0, y \in E(\xi) \\ 0 & \text { (otherwise) }\end{cases}$

Proof. First we prove (1). Let $E=E(\xi), C=E(\rho), A=O_{E}(-1)$ and $B=O_{C}(-1)$. Assume $E \neq C$. It suffices to consider the case $\{y\}=E \cap C$ by Lemma 4.5 . Hence $E C=1$. Since $y \in E$, we have an exact sequence

$$
\text { (u) } \quad: \quad 0 \rightarrow O_{E}(-1) \rightarrow O_{E} \rightarrow O_{y} \rightarrow 0
$$

which is a non-trivial extension given by a nonzero element of $\operatorname{Ext}_{O_{Y}}^{1}\left(O_{y}, A\right)$. Let $\gamma \in \operatorname{Hom}_{O_{Y}}\left(B, O_{y}\right)$ be a nonzero class. The cup product (4.4.1) is the pullback $\gamma^{*}(u)$ of $(u)$, which is given explicitly by

$$
0 \rightarrow O_{E}(-1) \rightarrow O_{E+C}(L) \rightarrow O_{C}(-1) \rightarrow 0
$$

where $L$ is the unique line bundle of $E+C$ such that $L_{E}=O_{E}$ and $L_{C}=$ $O_{C}(-1)$. This is a non-trivial extension because the following is non-trivial:

$$
0 \rightarrow O_{E}(-1) \rightarrow O_{E+C} \rightarrow O_{C} \rightarrow 0 .
$$

If $E=C$, then $\gamma^{*}(u)$ is trivial because $\operatorname{Ext}_{O_{Y}}^{1}(A, A)=0$ by Lemma 4.6.

\footnotetext{
${ }^{3} e=2$ in the $A_{1}$ case; otherwise $e \leq 1$.
} 
Next we prove (2). Since $\omega_{F} \cong K_{Y} \otimes_{O_{Y}} O_{F}(-F) \cong O_{F}(-F)$, we have $\operatorname{Hom}_{O_{Y}}\left(\omega_{F}, O_{E(\xi)}(-1)\right) \cong H^{0}\left(O_{E(\xi)}(-F E(\xi)-1)\right)$, which is non-zero if and only if $F E(\xi) \neq 0$ (note that $F E(\xi) \leq 0$ by the definition of the fundamental cycle). In this case, the evaluation map

$$
\operatorname{Hom}_{O_{Y}}\left(\omega_{F}, O_{E(\xi)}(-1)\right) \otimes_{k} \omega_{F} \rightarrow O_{E(\xi)}(-1)
$$

is surjective and therefore (2) is proved.

Summarizing Subsections 4.2-4.4, we obtain the following.

Theorem 4.8. Suppose $G \subset \mathrm{SL}(2)$. Under the above notation, let $y \in F$, $V(\xi) \subset \operatorname{Soc}\left(I_{y}\right)^{4}$ and $W(\rho) \subset \operatorname{Gen}\left(I_{y}\right)$. Then the following are equivalent:

(1) $W(\rho) \subset S_{1} \cdot V^{\natural}(\xi)$ in $\operatorname{Gen}\left(I_{y}\right)$,

(2) the extension (4.3.1) is non-trivial,

(3) the cup product (4.3.2) is nonzero,

(4) (4.4.1) is nonzero if $\rho \neq \rho_{0}$, while (4.4.2) is nonzero if $\rho=\rho_{0}$,

(5) $E(\xi) E(\rho)=1$ and $y \in E(\xi) \cap E(\rho)$ if $\rho \neq \rho_{0}$, while $E(\xi) F \neq 0{ }^{5}$ and $y \in E(\xi)$ if $\rho=\rho_{0}$.

See also Theorem 6.7 (1) for $G \not \subset \mathrm{SL}(2)$.

\section{Semi-Orthogonal projections}

5.1. Semi-orthogonal projection of $O_{0} \otimes_{k} \rho$. Let $A=D(Y), B=$ $D_{c}^{G}(U), F=\Phi_{O_{\mathcal{Z}}}$ and $H=\Phi_{O_{\mathcal{Z}}}^{*}$. We apply [4, Proposition 1.5] to them. Let $\operatorname{Im}(F)=\{b \in B ; b \simeq F a$ for some $a \in A\}$ and $\operatorname{Ker}(H)=\{c \in B ; H c \simeq 0\}$. By Corollary 3.17, $F=\Phi_{O_{z}}$ is fully faithful because $\Phi$ is fully faithful by Theorem 3.2. Moreover $F=\Phi_{O_{\mathcal{Z}}}: A \rightarrow B$ has a right adjoint $H=\Phi_{O \mathcal{Z}}^{*}: B \rightarrow A$ by Corollary 3.18. Hence $H F \simeq \mathrm{id}_{A}$. It follows that there is a semi-orthogonal decomposition $(\operatorname{Ker}(H), \operatorname{Im} F)$ of $B=D_{c}^{G}(U)$. Therefore $H F$ is the projection of $B$ to the subcategory $\operatorname{Im}(F)$, which we call a semi-orthogonal projection.

Let $\rho$ be an irreducible representation of $G$. In what follows we study the semi-orthogonal projection $H F=\Phi_{O_{\mathcal{Z}}} \circ \Phi_{O_{\mathcal{Z}}}^{*}$ of $O_{0} \otimes_{k} \rho$. In Subsection 5.1 we study the image by $H F$ of $O_{0} \otimes_{k} \rho$ for $\rho$ non-trivial special, while we study the image by $H F$ of $O_{0} \otimes_{k} \rho_{0}$ for $\rho_{0}$ trivial in Subsection 5.2. Note that if $\rho$ is non-special, this vanishes by Corollary 3.19.

In this subsection, we assume that $\rho$ is non-trivial special. We shall prove Theorem 5.7 analogous to [19, Theorem 7.2].

We quote [20, Lemma 2.4]:

Lemma 5.1. Let $f: Y \rightarrow X$ be a proper surjective morphism of surfaces with $X$ affine. Let $\mathcal{E}$ and $\mathcal{F}$ be coherent $O_{Y}$-modules. If $\mathcal{E}$ is generated by global sections and if $H^{1}(Y, \mathcal{F})=0$, then $H^{q}\left(Y, \mathcal{E} \otimes_{O_{Y}} \mathcal{F}\right)=0$ for $q>0$.

\footnotetext{
${ }^{4}$ hence $\xi \neq \rho_{0}$ by Theorem 3.6

$5_{\text {this }}$ is equivalent to $\xi=\rho_{\text {nat }}$ if it is not the $A_{n}$-case
} 
Corollary 5.2. Let $f: Y \rightarrow X$ be a minimal resolution of a normal affine surface $X$ with a rational singular point $P, F$ the fundamental divisor of $Y$ and $\mathcal{N}$ a coherent sheaf on $Y$. Then $H^{1}\left(Y, \mathcal{N} \otimes_{O_{Y}} O_{F}\right)=0$ if and only if $H^{1}(Y, \mathcal{N})=0$.

Proof. The proof is the same as in [18, Lemma 3.1], where we don't need the assumption that $\mathcal{E}$ (or $\mathcal{N}$ here) is locally free.

Lemma 5.3. If $H^{1}(Y, \mathcal{C})=0$, then $\Phi_{\mathcal{O}_{\mathcal{Z}}}(\mathcal{C})$ is a sheaf such that

$$
\pi_{*}\left(\Phi_{O_{\mathcal{Z}}}(\mathcal{C})\right) \simeq \bigoplus_{\sigma \in \operatorname{Irr}(G)} f_{*}\left(\mathcal{C} \otimes_{O_{Y}} \mathcal{M}_{\sigma}\right) \otimes_{k} \sigma
$$

Proof. By pushing forward to $X$, we obtain

$$
\begin{aligned}
\mathbf{R} \pi_{*}\left(\Phi_{O_{\mathcal{Z}}}(\mathcal{C})\right) & =\mathbf{R}\left(\pi \circ \pi_{U}\right)_{*}\left(\pi_{Y}^{*} \mathcal{C} \otimes_{O_{Y \times}{ }_{k}} O_{\mathcal{Z}}\right) \\
& \simeq \mathbf{R} f_{*}\left(\mathcal{C} \otimes_{O_{Y}} p_{*} O_{\mathcal{Z}}\right) \\
& \simeq \bigoplus_{\sigma \in \operatorname{Irr}(G)} \mathbf{R} f_{*}\left(\mathcal{C} \otimes_{O_{Y}} \mathcal{M}_{\sigma}\right) \otimes_{k} \sigma
\end{aligned}
$$

from Lemma 2.20. Since $\mathcal{M}_{\sigma}$ is a full $O_{Y}$-module, which is generated by global sections by definition, we have $H^{q}\left(\mathcal{C} \otimes_{O_{Y}} \mathcal{M}_{\sigma}\right)=0$ for $q>0$ by Lemma 5.1. Hence $\mathbf{R} \pi_{*}\left(\Phi_{O_{\mathcal{Z}}}(\mathcal{C})\right)=\pi_{*}\left(\Phi_{O_{\mathcal{Z}}}(\mathcal{C})\right)$ is a sheaf. This implies that $\Phi_{O_{\mathcal{Z}}}(\mathcal{C})$ is a sheaf because $\pi$ is an affine morphism.

Lemma 5.4. $\Phi_{O \mathcal{Z}}\left(\mathcal{M}_{\rho}^{\vee}\right) \simeq O_{U} \otimes_{k} \rho$ for $\rho \in \operatorname{Irr}(G)$ special.

Proof. Since $\rho$ is special, $\Phi_{O_{\mathcal{Z}}}\left(\mathcal{M}_{\rho}^{\vee}\right)$ is a sheaf by Lemma 5.3. Since $\mathcal{M}_{\sigma}$ is full for any $\sigma \in \operatorname{Irr}(G)$ by Lemma 2.20 (4), we have $H^{1}\left(\mathcal{M}_{\sigma}^{\vee} \otimes_{O_{Y}} \omega_{Y}\right)=0$. Hence by Lemma 5.1, $H^{1}\left(\mathcal{M}_{\rho} \otimes_{O_{Y}} \mathcal{M}_{\sigma}^{\vee} \otimes_{O_{Y}} \omega_{Y}\right)=0$. Therefore $\mathcal{M}_{\rho}^{\vee} \otimes_{O_{Y}} \mathcal{M}_{\sigma}$ satisfies the conditions (i) and (iii) of Lemma $2.3(1)$. Hence $f_{*}\left(\mathcal{M}_{\rho}^{\vee} \otimes O_{Y} \mathcal{M}_{\sigma}\right)$ is reflexive by Lemma $2.3(2)$. It follows that $\pi_{*}\left(\Phi_{O_{\mathcal{Z}}}\left(\mathcal{M}_{\rho}^{\vee}\right)\right)$ is a reflexive $O_{X}$-module, which is characterized by the vanishing

$$
0=H_{\{0\}}^{1}\left(X, \pi_{*}\left(\Phi_{O_{\mathcal{Z}}}\left(\mathcal{M}_{\rho}^{\vee}\right)\right)\right) \simeq H_{\{0\}}^{1}\left(U, \Phi_{O_{\mathcal{Z}}}\left(\mathcal{M}_{\rho}^{\vee}\right)\right)
$$

and therefore $\Phi_{O_{\mathcal{Z}}}\left(\mathcal{M}_{\rho}^{\vee}\right)$ is a reflexive $O_{U}$-module.

Since $U$ is regular, $\Phi_{O_{\mathcal{Z}}}\left(\mathcal{M}_{\rho}^{\vee}\right)$ is a locally free $O_{U}$-module by Lemma 2.2 (2). It follows from (5.1.1), Lemma 2.20 and Corollary 2.13 that

$$
\pi_{*}\left(\Phi_{O_{\mathcal{Z}}}\left(\mathcal{M}_{\rho}^{\vee}\right)\right)^{G} \simeq \bigoplus_{\sigma \in \operatorname{Irr}(G)}\left(f_{*}\left(\mathcal{M}_{\rho}^{\vee} \otimes_{O_{Y}} \mathcal{M}_{\sigma}\right) \otimes_{k} \sigma\right)^{G}=f_{*}\left(\mathcal{M}_{\rho}^{\vee}\right) \simeq M_{\rho^{*}}
$$

By Lemma 2.11, $\Phi_{O_{\mathcal{Z}}}\left(\mathcal{M}_{\rho}^{\vee}\right)$ is uniquely determined by the reflexive module $\pi_{*}\left(\Phi_{O_{\mathcal{Z}}}\left(\mathcal{M}_{\rho}^{\vee}\right)\right)^{G}$. Since $\left(O_{U} \otimes_{k} \rho\right)^{G}=M_{\rho^{*}}$, we obtain the assertion. 
Definition 5.5. For special representations $\rho$ and $\sigma$, we define a nonnegative integer $a_{\rho \sigma}$ as follows:

$$
a_{\rho \sigma}= \begin{cases}E(\rho) E(\sigma) & \rho, \sigma \text { non-trivial, } \rho \neq \sigma \\ -F E(\rho) & \rho \neq \rho_{0}, \sigma=\rho_{0} \\ \max \left\{-\left(K_{Y}+F\right) E(\rho), 0\right\} & \rho=\rho_{0}, \sigma \neq \rho_{0} \\ 0 & \rho=\sigma\end{cases}
$$

Proposition 5.6. Let $\rho \in \operatorname{Irr}(G)$ be non-trivial special, and $E(\rho)$ the corresponding exceptional curve. Then, the following hold:

(1) $\Phi_{O_{\mathcal{Z}}}\left(O_{E(\rho)}(-1)\right)$ is a quotient sheaf of $O_{U} \otimes_{k} \rho$,

(2) let $J(\rho)$ be the kernel of the surjection $O_{U} \otimes_{k} \rho \rightarrow \Phi_{O_{\mathcal{Z}}}\left(O_{E(\rho)}(-1)\right)$. Then

$$
\begin{aligned}
J(\rho) / \mathfrak{m} J(\rho) & \simeq \underset{\sigma \in \operatorname{Irr}(G): \text { special }}{\bigoplus} \sigma^{\oplus a_{\rho, \sigma}}, \\
O_{U} \otimes_{k} \rho / J(\rho) & \simeq \Phi_{O_{\mathcal{Z}}}\left(O_{E(\rho)}(-1)\right),
\end{aligned}
$$

(3) $J(\rho)$ is the $O_{U}$-submodule of $O_{U} \otimes_{k} \rho$ generated by all the special representations in $\mathfrak{m} \otimes_{k} \rho$.

Proof. By Lemma 2.3 and Theorem 2.8, there is an isomorphism

$$
\left.\mathcal{M}_{\rho}\right|_{E(\rho)} \simeq O_{E(\rho)}(1) \oplus O_{E(\rho)}^{\oplus \operatorname{dim} \rho-1} .
$$

Hence there is a surjection $j(\rho): \mathcal{M}_{\rho}^{\vee} \rightarrow O_{E(\rho)}(-1)$. Let $\mathcal{K}_{\rho}=\operatorname{Ker}(j(\rho))$. Then there is an exact sequence

$$
0 \rightarrow \mathcal{K}_{\rho} \rightarrow \mathcal{M}_{\rho}^{\vee} \rightarrow O_{E(\rho)}(-1) \rightarrow 0
$$

Since $\rho$ is special, $H^{1}\left(\mathcal{M}_{\rho}^{\vee}\right)=0$. Hence $H^{1}\left(Y, \mathcal{K}_{\rho}\right)=0$ by (5.1.3). Thus Lemma 5.3 implies that any of $\Phi_{O_{\mathcal{Z}}}\left(\mathcal{K}_{\rho}\right), \Phi_{O_{\mathcal{Z}}}\left(\mathcal{M}^{\vee}\right)$ and $\Phi_{O_{\mathcal{Z}}}\left(O_{E(\rho)}(-1)\right)$ is a sheaf at degree 0. Applying $\Phi_{O_{\mathcal{Z}}}$ to (5.1.3), we obtain a distinguished triangle

$$
\Phi_{O_{\mathcal{Z}}}\left(\mathcal{K}_{\rho}\right) \rightarrow \Phi_{O_{\mathcal{Z}}}\left(\mathcal{M}_{\rho}^{\vee}\right) \rightarrow \Phi_{O_{\mathcal{Z}}}\left(O_{E(\rho)}(-1)\right) \rightarrow \Phi_{O_{\mathcal{Z}}}\left(\mathcal{K}_{\rho}\right)[1],
$$

which reduces to a short exact sequence of sheaves

$$
0 \rightarrow \Phi_{O_{\mathcal{Z}}}\left(\mathcal{K}_{\rho}\right) \rightarrow \Phi_{O_{\mathcal{Z}}}\left(\mathcal{M}_{\rho}^{\vee}\right) \rightarrow \Phi_{O_{\mathcal{Z}}}\left(O_{E(\rho)}(-1)\right) \rightarrow 0 .
$$

Thus $\Phi_{O_{\mathcal{Z}}}\left(O_{E(\rho)}(-1)\right)$ is a quotient sheaf of $\Phi_{O_{\mathcal{Z}}}\left(\mathcal{M}_{\rho}^{\vee}\right) \simeq O_{U} \otimes_{k} \rho$ by Lemma 5.4. Hence (1) is proved.

Let $\sigma$ be an irreducible representation of $G$. Then the multiplicity of $\sigma$ in $J(\rho) / \mathfrak{m} J(\rho)$ is given by the dimension of

$$
\begin{aligned}
\operatorname{Ext}^{1}\left(O_{U} \otimes_{k} \rho / J(\rho), O_{0} \otimes_{k} \sigma\right) & =\operatorname{Ext}^{1}\left(\Phi_{O_{\mathcal{Z}}}\left(O_{E(\rho)}(-1)\right), O_{0} \otimes_{k} \sigma\right) \\
& \simeq \operatorname{Ext}^{1}\left(O_{E(\rho)}(-1), \Phi_{O_{\mathcal{Z}}}^{*}\left(O_{0} \otimes_{k} \sigma\right)\right) .
\end{aligned}
$$


If $\sigma$ is non-special, then this vanishes by Corollary 3.19. If $\sigma$ is special nontrivial, $\operatorname{dim} \operatorname{Ext}^{1}\left(O_{E(\rho)}(-1), \Phi_{O_{\mathcal{Z}}}^{*}\left(O_{0} \otimes_{k} \sigma\right)\right)=a_{\rho, \sigma}$ follows from Corollary 3.19 and Lemma 4.6. If $\sigma=\rho_{0}$, then by Corollary 3.19

$$
\begin{aligned}
\operatorname{Ext}^{1}\left(O_{E(\rho)}(-1), \Phi_{O_{\mathcal{Z}}}^{*}\left(O_{0} \otimes_{k} \sigma\right)\right) & \simeq \operatorname{Ext}^{1}\left(O_{E(\rho)}(-1), \omega_{F}[1]\right) \\
& \simeq \operatorname{Ext}^{2}\left(O_{E(\rho)}(-1),\left(\omega_{Y}+F\right) \otimes_{O_{Y}} O_{F}\right) \\
& \simeq \operatorname{Ext}^{0}\left(O_{F}(F), O_{E(\rho)}(-1)\right)^{\vee} \\
& \simeq \operatorname{Ext}^{0}\left(O_{F}, O_{E(\rho)}(-1-F E(\rho))\right)^{\vee},
\end{aligned}
$$

whose dimension is equal to $a_{\rho, \rho_{0}}$. Hence we derive $(2)$.

Finally we shall prove (3). If $\sigma=\rho$, then $H^{0}\left(\mathcal{M}_{\rho} \otimes_{O_{Y}} O_{E(\rho)}(-1)\right) \simeq k$ by (5.1.2). Meanwhile for any special $\sigma(\neq \rho)$, we have $\left.\mathcal{M}_{\sigma}\right|_{E(\rho)} \simeq O_{E(\rho)}^{\oplus \operatorname{dim} \sigma}$ and $H^{0}\left(\mathcal{M}_{\rho} \otimes_{O_{Y}} O_{E(\rho)}(-1)\right)=0$. By Lemma 5.3

$$
\pi_{*} \Phi_{O_{\mathcal{Z}}}\left(O_{E(\rho)}(-1)\right) \simeq \rho \oplus \underset{\sigma \in \operatorname{Irr}(G): \text { non-special }}{\bigoplus} H^{0}\left(\mathcal{M}_{\sigma} \otimes_{O_{Y}} O_{E(\rho)}(-1)\right) \otimes_{k} \sigma .
$$

Therefore $\left(\mathfrak{m} \otimes_{k} \rho\right) / J(\rho)$ contains no special representations. Since $J(\rho)$ is generated by special representations by (2), we obtain (3).

By [19, Theorem 7.2], $y \in E(\rho)$ if and only if $\rho \otimes \rho_{\operatorname{det}} \subset \operatorname{Soc}\left(O_{Z_{y}}\right)$. Corollary 2.17 shows that, if $G \not \subset \mathrm{SL}(2, k)$, and if $\rho$ is non-trivial and special, then $\rho \otimes \rho_{\text {det }}$ is non-special. In contrast, Theorem 5.7 characterizes $y \in E(\rho)$ by the existence of a certain $k[G]$-submodule $(\simeq \rho)$ of $O_{Z_{y}}$, which is regarded as a natural generalization of a socle of $O_{Z_{y}}$.

Theorem 5.7. Let $\rho \in \operatorname{Irr}(G)$ be non-trivial special. For $y \in F$, the following conditions are equivalent:

(1) $y \in E(\rho)$.

(2) There is a $k[G]$-submodule $V(\rho) \subset O_{Z_{y}}$ isomorphic to $\rho$ such that the $O_{U}$-submodule of $O_{Z_{y}}$ generated by $V(\rho)$ contains no other special representations.

Moreover, $V(\rho)$ is unique if it exists.

Proof. Since $\Phi_{O_{\mathcal{Z}}}$ is fully faithful, we have by $\Phi_{O_{\mathcal{Z}}}\left(O_{y}\right)=O_{Z_{y}}$

$$
\begin{aligned}
\operatorname{Hom}_{D^{G}(U)} & \left(O_{U} \otimes_{k} \rho / J(\rho), O_{Z_{y}}\right) \\
& \simeq \operatorname{Hom}_{D^{G}(U)}\left(\Phi_{O_{\mathcal{Z}}}\left(O_{E(\rho)}(-1)\right), \Phi_{O_{\mathcal{Z}}}\left(O_{y}\right)\right) \\
& \simeq \operatorname{Hom}_{D(Y)}\left(O_{E(\rho)}(-1), O_{y}\right) .
\end{aligned}
$$

Assume $y \in E(\rho)$. By (5.1.5) the inclusion $\{y\} \hookrightarrow E(\rho)$ induces a nonzero $G$-homomorphism

$$
\zeta_{y}: O_{U} \otimes_{k} \rho / J(\rho) \rightarrow O_{Z_{y}} .
$$

Let $V(\rho)$ be the image of the $k[G]$-submodule $k \otimes_{k} \rho$ of $O_{U} \otimes_{k} \rho / J(\rho)$ by $\zeta_{y}$. Since $k \otimes_{k} \rho$ generates $O_{U} \otimes_{k} \rho / J(\rho)$, it is clear that $V(\rho) \simeq \rho$. Then 
(2) follows from Proposition 5.6 (3). Since (5.1.5) is one-dimensional, $V(\rho)$ is unique.

Conversely suppose that (2) is true. Any $k[G]$-isomorphism $k \otimes_{k} \rho \simeq$ $V(\rho)$ extends to an $O_{U}$-homomorphism from $O_{U} \otimes_{k} \rho$ to $O_{Z_{y}}$ uniquely, which annihilates $J(\rho)$ by Proposition $5.6(3)$ and therefore induces an $O_{U^{-}}$ homomorphism from $O_{U} \otimes_{k} \rho / J(\rho)$ to $O_{Z_{y}}$. Hence it induces a non-zero $O_{Y}$-homomorphism from $O_{E(\rho)}(-1)$ to $O_{y}$ by (5.1.5), so $y \in E(\rho)$. This proves (1).

Definition 5.8. We call the submodule $O_{U} V(\rho) \subset O_{Z_{y}}$ the mono-special $O_{U}$-submodule of $O_{Z_{y}}$ (associated to $\rho$ ).

Note that no socle of $O_{Z_{y}}$ is a mono-special $O_{U}$-submodule of $O_{Z_{y}}$ if $G \not \subset \mathrm{SL}(2)$. If $G \subset \mathrm{SL}(2), V(\rho)$ is a socle of $O_{Z_{y}}$ if and only if it is a mono-special $O_{U}$-submodule of $O_{Z_{y}}$.

Assume $y \in E(\rho)$. Then by (5.1.6) $\zeta_{y}$ induces an $O_{U}$-homomorphism

$$
\alpha_{y}: O_{U} \otimes_{k} \rho \rightarrow O_{Z_{y}}
$$

sending $k \otimes_{k} \rho \subset O_{U} \otimes_{k} \rho$ to $V(\rho)$. Then $J(\rho)$ can be described as follows.

Lemma 5.9. $J(\rho)=\bigcap_{y \in E(\rho)} \operatorname{ker} \alpha_{y}$ for $\rho \in \operatorname{Irr}(G)$ non-trivial special.

Proof. The inclusion $J(\rho) \subset \operatorname{ker} \alpha_{y}$ is obvious. Decompose $\alpha_{y}$ as

$$
O_{U} \otimes_{k} \rho \rightarrow O_{U} \otimes_{k} \rho / J(\rho) \simeq \Phi_{O_{\mathcal{Z}}}\left(O_{E(\rho)(-1)}\right) \stackrel{\eta_{y}}{\rightarrow} \Phi_{O_{\mathcal{Z}}}\left(O_{y}\right) \simeq O_{Z_{y}} .
$$

where $\eta_{y}$ is the restriction map induced from the inclusion $\{y\} \hookrightarrow E(\rho)$.

We recall by Lemma 5.3

$$
\begin{aligned}
\pi_{*} \Phi_{O_{\mathcal{Z}}}\left(O_{E(\rho)}(-1)\right) & =\bigoplus_{\sigma \in \operatorname{Irr}(G)} H^{0}\left(\mathcal{M}_{\sigma} \otimes_{O_{Y}} O_{E(\rho)}(-1)\right) \otimes_{k} \sigma \\
\pi_{*} \Phi_{O_{\mathcal{Z}}}\left(O_{y}\right) & =\bigoplus_{\sigma \in \operatorname{Irr}(G)} H^{0}\left(\mathcal{M}_{\sigma} \otimes_{O_{Y}} O_{y}\right) \otimes_{k} \sigma
\end{aligned}
$$

Let $W=\bigcap_{y \in E(\rho)}$ ker $\eta_{y}$. To prove the assertion, it suffices to check $W=0$. Let $\phi=\sum_{\sigma \in \operatorname{Irr}(G)} \phi_{\sigma} \otimes \sigma \in W$ for $\phi_{\sigma} \in H^{0}\left(\mathcal{M}_{\sigma} \otimes_{O_{Y}} O_{E(\rho)}(-1)\right)$. Then the evaluation of $\phi_{\sigma}$ at every $y \in E(\rho)$ is equal to zero, hence $\phi_{\sigma}$ is zero because $\mathcal{M}_{\sigma} \otimes_{O_{Y}} O_{E(\rho)}(-1)$ is locally $O_{E(\rho)}$-free. Hence $\phi=0$ so that $W=0$. This completes the proof.

5.2. Semiorthogonal projection of $O_{0} \otimes_{k} \rho_{0}$. In this subsection, we consider $\Phi_{O_{\mathcal{Z}}}\left(\omega_{F}[1]\right)=\Phi_{O_{\mathcal{Z}}} \circ \Phi_{O_{\mathcal{Z}}}^{*}\left(O_{0} \otimes_{k} \rho_{0}\right)$.

Lemma 5.10. Let $\Phi_{O_{\mathcal{Z}}}^{i}\left(\omega_{F}[1]\right)$ be the $i$-th cohomology sheaf of $\Phi_{O_{\mathcal{Z}}}\left(\omega_{F}[1]\right)$. Then $\Phi_{O_{\mathcal{Z}}}^{i}\left(\omega_{F}[1]\right)$ is a $k[G]$-module such that:

(1) $\Phi_{O_{\mathcal{Z}}}^{i}\left(\omega_{F}[1]\right)=0$ if $i \neq 0,-1$,

(2) $\Phi_{O_{\mathcal{Z}}}^{0}\left(\omega_{F}[1]\right)$ contains $\rho_{0}$ with multiplicity one and it contains no other special representations, 
(3) $\Phi_{O_{\mathcal{Z}}}^{(-1)}\left(\omega_{F}[1]\right)$ contains no special representations, where $\Phi_{O_{\mathcal{Z}}}^{(-1)}\left(\omega_{F}[1]\right)=$ 0 if and only if $G \subset \mathrm{SL}(2, k)$.

Proof. By (5.1.1), we have $\Phi_{O \mathcal{Z}}^{i}\left(\omega_{F}[1]\right)=\Phi_{O \mathcal{Z}}^{i+1}\left(\omega_{F}\right)=0$ for $i \neq-1,0$, hence (1) is proved.

By the Serre duality for $F$, we have

$$
\begin{aligned}
\pi_{*} \Phi_{O_{\mathcal{Z}}}^{i}\left(\omega_{F}[1]\right) & =\bigoplus_{\rho \in \operatorname{Irr}(G)} H^{i+1}\left(\mathcal{M}_{\rho} \otimes_{O_{Y}} \omega_{F}\right) \otimes_{k} \rho \\
& \simeq \bigoplus_{\rho \in \operatorname{Irr}(G)} H^{-i}\left(\mathcal{M}_{\rho}^{\vee} \otimes_{O_{Y}} O_{F}\right)^{\vee} \otimes_{k} \rho .
\end{aligned}
$$

Now we shall prove (2). The multiplicity of the trivial representation $\rho_{0}$ in $\Phi_{O_{\mathcal{Z}}}^{0}\left(\omega_{F}[1]\right)$ is equal to $\operatorname{dim} H^{0}\left(\mathcal{M}_{\rho_{0}}^{\vee} \otimes_{O_{Y}} O_{F}\right)=\operatorname{dim} H^{0}\left(O_{F}\right)=1$ by $[1$, Theorem 4, p. 132]. We note that $H^{1}\left(O_{F}\right)=0$ so that $\chi\left(O_{F}\right)=1$. Let $\rho$ be a non-trivial special representation. Then $\operatorname{rank}\left(\mathcal{M}_{\rho}\right)=c_{1}\left(\mathcal{M}_{\rho}\right) F$ by Theorem 2.8 (2) (see [33]). Then by Riemann-Roch,

$$
\chi\left(\mathcal{M}_{\rho}^{\vee} \otimes_{O_{Y}} O_{F}\right)=-c_{1}\left(\mathcal{M}_{\rho}\right) F+\left(\operatorname{rank}\left(\mathcal{M}_{\rho}\right)\right) \chi\left(O_{F}\right)=0 .
$$

By $H^{1}\left(\mathcal{M}_{\rho}^{\vee} \otimes_{O_{Y}} O_{F}\right)=0$, we have $H^{0}\left(\mathcal{M}_{\rho}^{\vee} \otimes_{O_{Y}} O_{F}\right)=0$. This proves $(2)$.

Next we shall prove (3). By Corollary 5.2, we see the following:

- $H^{1}\left(\mathcal{M}_{\rho}^{\vee} \otimes_{O_{Y}} O_{F}\right)=0$ if and only if $\rho$ is special, that is, $H^{1}\left(\mathcal{M}_{\rho}^{\vee}\right)=0$,

$-H^{1}\left(\mathcal{M}_{\rho}^{\vee} \otimes_{O_{Y}} O_{F}\right) \neq 0$ if and only if $\rho$ is non-special.

Hence (5.2.1) implies

- there is no $\rho$-part of $\pi_{*} \Phi_{O \mathcal{Z}}^{(-1)}\left(\omega_{F}[1]\right)$ for $\rho$ special,

- the $\rho$-part of $\pi_{*} \Phi_{O_{\mathcal{Z}}}^{(-1)}\left(\omega_{F}[1]\right)$ is non-zero for $\rho$ non-special.

Therefore, $\Phi_{O \mathcal{Z}}^{(-1)}\left(\omega_{F}[1]\right) \neq 0$ if and only if there is a non-special representation of $G$, which is equivalent to $G \not \subset \mathrm{SL}(2, k)$ by Corollary 2.17. This proves (3).

Proposition 5.11. For $\rho \in \operatorname{Irr}(G)$, we have

$$
\begin{aligned}
& \operatorname{dim} \operatorname{Ext}_{D_{c}^{G}(U)}^{p}\left(\Phi_{O_{\mathcal{Z}}}^{0}\left(\omega_{F}[1]\right), O_{0} \otimes_{k} \rho\right) \\
& \quad= \begin{cases}1 & \text { if } p=0, \rho=\rho_{0}, \\
\max \left\{-\left(K_{Y}+F\right) E(\rho), 0\right\} & \text { if } p=1, \rho: \text { non-trivial special, } \\
0 & \text { otherwise. }\end{cases}
\end{aligned}
$$

Proof. For a fixed $\rho \in \operatorname{Irr}(G)$, we consider the spectral sequence

$$
E_{2}^{p, q}=\operatorname{Ext}_{D_{c}^{G}(U)}^{p}\left(\Phi_{O_{\mathcal{Z}}}^{(-q)}\left(\omega_{F}[1]\right), O_{0} \otimes_{k} \rho\right)
$$

with abutment

$$
E_{\infty}^{p+q}=\operatorname{Hom}_{D_{c}^{G}(U)}^{p+q}\left(\Phi_{O_{\mathcal{Z}}}\left(\omega_{F}[1]\right), O_{0} \otimes_{k} \rho\right) .
$$


Since the global dimension of $\mathrm{Coh}^{G} U$ is 2 , we have $E_{2}^{p, q}=0$ unless $0 \leq p \leq 2$ and $0 \leq q \leq 1$. Then the standard property of the spectral sequence implies $E_{2}^{0,0}=E_{\infty}^{0}$ and $E_{2}^{1,0} \subset E_{\infty}^{1,0} \subset E_{\infty}^{1}$.

Now we compute $E_{\infty}^{i}(i=0,1)$. By Corollary 3.19,

$$
\begin{aligned}
E_{\infty}^{p} & \simeq \operatorname{Hom}_{D(Y)}^{p}\left(\omega_{F}[1], \Phi_{O_{\mathcal{Z}}}^{*}\left(O_{0} \otimes_{k} \rho\right)\right) \\
& \simeq \begin{cases}\operatorname{Hom}_{D(Y)}^{p}\left(\omega_{F}[1], \omega_{F}[1]\right) & \text { if } \rho=\rho_{0} \\
\operatorname{Hom}_{D(Y)}^{p-1}\left(\omega_{F}, O_{E(\rho)}(-1)\right) & \text { if } \rho \text { is non-trivial special } \\
0 & \text { if } \rho \text { is nonspecial. }\end{cases}
\end{aligned}
$$

For $\rho=\rho_{0}$, we have $E_{\infty}^{0} \simeq H^{0}\left(O_{F}\right) \simeq k$ and $E_{\infty}^{1} \simeq \operatorname{Ext}_{O_{Y}}^{1}\left(O_{F}, O_{F}\right)=0$. If $\rho$ is non-trivial special, then $E_{\infty}^{0}=0$ and $E_{\infty}^{1} \simeq H^{1}\left(O_{E(\rho)}(-1) \otimes_{O_{F}} \omega_{F}^{-1}\right)$. This completes the proof.

Corollary 5.12. The following holds:

1. $\Phi_{O_{\mathcal{Z}}}^{0}\left(\omega_{F}[1]\right)$ is a quotient sheaf of $O_{U} \otimes_{k} \rho_{0}$.

2. Let $J\left(\rho_{0}\right)$ be the kernel of the surjection $O_{U} \otimes_{k} \rho_{0} \rightarrow \Phi_{O \mathcal{Z}}^{0}\left(\omega_{F}[1]\right)$. Then

- $J\left(\rho_{0}\right)$ contains all special representations in $\mathfrak{m} \otimes_{k} \rho_{0}$,

- the minimal generators of $J\left(\rho_{0}\right)$ are all non-trivial special representations $\sigma$ in $\mathfrak{m} \otimes_{k} \rho_{0}$ with $\left(K_{Y}+F\right) E(\sigma)<0$, each with multiplicity $-\left(K_{Y}+F\right) E(\sigma)$.

Proof. Let $\mathcal{F}=\Phi^{0}\left(\omega_{F}[1]\right)$. Proposition 5.11 shows $\mathcal{F} / \mathfrak{m} \mathcal{F} \simeq \rho_{0}$, which implies that $\mathcal{F}$ is generated over $O_{U}$ by a single element of $\mathcal{F}$ since $\mathcal{F}$ is supported by the origin of $U$. In other words, there is a surjection $f$ : $O_{U} \otimes \rho_{0} \rightarrow \mathcal{F}$. This proves $(1)$. Let $J\left(\rho_{0}\right)=\operatorname{ker}(f)$. Then there is an exact sequence

$$
0 \rightarrow J\left(\rho_{0}\right) \rightarrow O_{U} \otimes_{k} \rho_{0} \rightarrow \mathcal{F} \rightarrow 0,
$$

from which we infer $\operatorname{Ext}_{D^{G}(U)}^{1}\left(\mathcal{F}, O_{0} \otimes_{k} \rho_{0}\right) \simeq \operatorname{Hom}_{D^{G}(U)}\left(J\left(\rho_{0}\right), O_{0} \otimes_{k} \rho_{0}\right)$ because $\operatorname{Ext}_{D^{G}(U)}^{1}\left(O_{U}, O_{0} \otimes_{k} \rho_{0}\right)=H^{1}\left(U, O_{0} \otimes_{k} \rho_{0}\right)^{G}=0$. Thus (2) follows from Lemma 5.10 (2), Proposition 5.11 and

$$
\operatorname{Hom}_{D^{G}(U)}\left(J\left(\rho_{0}\right), O_{0} \otimes_{k} \rho_{0}\right) \simeq \operatorname{Hom}_{D^{G}(U)}\left(J\left(\rho_{0}\right) / \mathfrak{m} J\left(\rho_{0}\right), O_{0} \otimes_{k} \rho_{0}\right) .
$$

Proposition 5.6 (2) and Corollary 5.12 are summarized as follows:

Corollary 5.13. For $\rho \in \operatorname{Irr}(G)$ special, there is an $k[G]$-isomorphism

$$
J(\rho) / \mathfrak{m} J(\rho) \simeq \bigoplus_{\sigma \in \operatorname{Irr}(G): \text { special }} \sigma^{\oplus a_{\rho \sigma}}
$$




\section{Reconstruction algebra and deformations of $G$-Clusters}

\subsection{The reconstruction algebra of $X$. Let}

$$
E=\bigoplus_{\rho: \text { special }} O_{U} \otimes_{k} \rho
$$

An endomorphism of $E$ is defined to be a $G$-equivariant $O_{U}$-linear endomorphism of $E$. The purpose of this subsection is to study the endomorphism algebra End $(E)$ of $E$ and briefly recall the reconstruction algebra of $X$ due to Wemyss.

Remark 6.1. Let $\rho$ and $\sigma \in \operatorname{Irr}(G)$ be both special. By Corollary 5.13 we choose $G$-equivariant $O_{U}$-homomorphisms,

$$
\phi_{\rho, \sigma}^{i}: O_{U} \otimes_{k} \sigma \rightarrow \mathfrak{m} \otimes_{k} \rho \subset O_{U} \otimes_{k} \rho \quad\left(1 \leq i \leq a_{\rho \sigma}\right)
$$

which are lifts of linearly independent $G$-homomorphisms from $O_{U} \otimes_{k} \sigma$ to $J(\rho) / \mathfrak{m} J(\rho)$. We may assume each $\phi_{\rho, \sigma}^{i}$ is a homogeneous $O_{U}$-homomorphism (that is, a homomorphism defined by homogeneous polynomials) because the diagonal $\mathbf{G}_{m}$ action on $U$ commutes with the action of $G$, hence it lifts to the actions on $X$ and $Y$, therefore every homogeneous term of $\phi_{\rho, \sigma}^{i}$ is also a homomorphism from $O_{U} \otimes_{k} \sigma$ to $\mathfrak{m} \otimes_{k} \rho \subset O_{U} \otimes_{k} \rho$.

We define an $O_{U}$-homomorphism

$$
\widetilde{\alpha}: \bigoplus_{\tau: \text { special }} O_{U} \otimes_{k} \tau \rightarrow J(\rho)
$$

by $\widetilde{\alpha}=\sum_{\tau} \phi_{\rho, \tau}^{i}$. Then by Nakayama's lemma, $\widetilde{\alpha}$ is surjective.

Proposition 6.2. Let $E=E_{X}:=\bigoplus_{\rho: \text { special }} O_{U} \otimes_{k} \rho$ and $X=U / G$. Then End $(E)$ is an $O_{X}$-algebra generated by $\phi_{\rho, \sigma}^{i}$ for $1 \leq i \leq a_{\rho \sigma}$ and the identity morphism of $O_{U} \otimes_{k} \rho$ where $\rho, \sigma$ range over the set of all special representations of $G$.

Proof. Let $\mathcal{A}$ be the subalgebra of $\bigoplus_{\rho \text { :special }} O_{U} \otimes_{k} \rho$ generated by $\left\{\phi_{\rho, \sigma}^{i}\right\}$ together with the identity morphisms of $O_{U} \otimes_{k} \rho$. Let $f: O_{U} \otimes_{k} \sigma \rightarrow O_{U} \otimes_{k} \rho$ be a non-zero homomorphism. We prove by the induction on $\operatorname{deg} f$ that if $f$ is homogeneous then $f \in \mathcal{A}$. If $\operatorname{deg} f=0$, then $\sigma=\rho$ and $f$ is a scalar multiple of the identity of $O_{U} \otimes_{k} \rho$. If $\operatorname{deg} f>0$, then $f$ factors through $J(\rho)$. By Remark 6.1, $\widetilde{\alpha}$ is surjective. Since $O_{U} \otimes_{k} \sigma$ is projective as an $O_{U^{-}}$ module with $G$-action ${ }^{6}$, there exists a $G$-equivariant $O_{U}$-homomorphism $\psi: O_{U} \otimes_{k} \sigma \rightarrow \oplus_{\tau: \text { special }} O_{U} \otimes_{k} \tau$ such that $f=\widetilde{\alpha} \circ \psi$. Let $\psi=\oplus_{\tau \text { :special }} f_{\tau}^{i}$, where $f_{\tau}^{i}: O_{U} \otimes_{k} \sigma \rightarrow O_{U} \otimes_{k} \tau$ is a $G$-equivariant $O_{U}$-homomorphism, homogeneous of degree $\operatorname{deg} f-\operatorname{deg} \phi_{\rho, \tau}^{i}<\operatorname{deg} f$. Then $f$ is expressed as

$$
f=\sum_{i ; \tau: \text { special }} \phi_{\rho, \tau}^{i} f_{\tau}^{i} \text {. }
$$

By the induction hypothesis, $f_{\tau}^{i} \in \mathcal{A}$ and hence $f \in \mathcal{A}$.

\footnotetext{
${ }^{6}$ because $|G|$ is prime to the characteristic of $k$
} 
Definition 6.3. Following Craw [7] and Wemyss [29], we consider the following quiver $Q=Q_{X}$ :

- the vertices of $Q$ are the special representations and

- the number of arrows from $\rho$ to $\sigma$ is $a_{\rho \sigma}$.

Following Craw [7] we call it the special McKay quiver of $X$.

Definition 6.4. Let $k Q$ be the path algebra of $Q=Q_{X}$. Proposition 6.2 shows that there is an isomorphism

$$
\operatorname{End}\left(\bigoplus_{\rho: \text { special }} O_{U} \otimes_{k} \rho\right) \simeq k Q / \mathcal{I},
$$

where $k Q$ is an $O_{X}$-algebra, and $\mathcal{I}$ is a certain two-sided ideal of $k Q$. The $O_{X}$-algebra End $\left(E_{X}\right)$ is called the reconstruction algebra of $X$.

Remark 6.5. The generators of the ideal $\mathcal{I}$ (in the completion $\widehat{k Q}$ ) are given explicitly in the cases of type $\mathrm{A}$ and type $\mathrm{D}$ by Wemyss [30, 31, 32].

6.2. G-clusters. In this subsection, we generalize Theorem 4.8.

As before we use the following notation:

$$
\operatorname{Ext}^{k}(A, B):=\operatorname{Hom}_{D(Y)}^{k}(A, B), \quad \operatorname{Ext}^{k}(C, D):=\operatorname{Hom}_{D^{G}(U)}^{k}(C, D),
$$

for $A, B \in D(Y)$ and $C, D \in D^{G}(U)$.

For $y \in E(\rho)$, recall that there are subrepresentations $W(\rho) \subset \operatorname{Gen}\left(I_{y}\right)$ and $W\left(\rho_{0}\right) \subset \operatorname{Gen}\left(I_{y}\right)$ isomorphic to $\rho$ and $\rho_{0}$ respectively. Moreover, there is a mono-special $O_{U}$-submodule $O_{U} V(\rho)$ of $O_{Z_{y}}$ by Theorem 5.7.

Definition 6.6. Let $\operatorname{SSoc}\left(O_{Z_{y}}\right)$ be the direct sum of $k[G]$-submodules $V(\rho) \subset$ $O_{Z_{y}}$ generating mono-special $O_{U}$-submodules with $y \in E(\rho)$.

Take any $k[G]$-submodule $\widetilde{V}(\rho) \subset O_{U}$ which is a lift of $V(\rho)$.

Theorem 6.7. Let $\rho$ and $\sigma$ be special representations, and $\rho \neq \sigma$.

(1) Suppose $\rho$ and $\sigma$ are non-trivial. Then $a_{\rho \sigma}$ is equal to the number of points $y \in F$ such that

- $W(\sigma) \subset \operatorname{Gen}\left(I_{y}\right)$,

$-V(\rho) \subset \operatorname{SSoc}\left(O_{Z_{y}}\right)$ and

- W( $\sigma) \subset\left(\mathfrak{m} \widetilde{V}(\rho)+\mathfrak{m} I_{y}\right) / \mathfrak{m} I_{y}$.

Actually, $a_{\rho \sigma}$ is either 0 or 1 and $a_{\rho \sigma}=1$ if and only if $E(\rho) \cap E(\sigma)$ consists of a unique point $y$.

(2) Suppose $\sigma=\rho_{0}$. Then, $a_{\rho \sigma} \neq 0$ if and only if there exists $y \in F$ such that

- $W(\sigma) \subset \operatorname{Gen}\left(I_{y}\right)$,

$-V(\rho) \subset \operatorname{SSoc}\left(O_{Z_{y}}\right)$, and

- $W(\sigma) \subset\left(\mathfrak{m} \widetilde{V}(\rho)+\mathfrak{m} I_{y}\right) / \mathfrak{m} I_{y}$.

If this holds for one $y$, then it holds for any $y \in E(\rho){ }^{7}$

\footnotetext{
${ }^{7} W(\sigma)$ depends on $y$. See Subsection 7.2.
} 
(3) Suppose $\rho=\rho_{0}$ and $\sigma \neq \rho_{0}$. Then $a_{\rho \sigma} \leq 2$, equality holding if and only if $F=E(\sigma)$, and $G$ is a cyclic group $\left\langle\frac{1}{n}(1,1)\right\rangle$. Moreover,

(3a) if $\operatorname{dim} \sigma=1$, then $a_{\rho \sigma}$ is described as follows:

$$
a_{\rho \sigma}= \begin{cases}0 & \text { if } W(\sigma) \subset \mathfrak{m} J\left(\rho_{0}\right) / \mathfrak{m} I_{y} \text { for any } y \in E(\sigma) \\ 2 & \text { if } W(\sigma) \not \subset \mathfrak{m} J\left(\rho_{0}\right) / \mathfrak{m} I_{y} \text { for any } y \in E(\sigma) \\ 1 & \text { otherwise. }\end{cases}
$$

(3b) if $\operatorname{dim} \sigma>1$, then $a_{\rho \sigma}$ is either 0 or 1 . The equality $a_{\rho \sigma}=1$ holds if and only if there is a $G$-invariant ideal $J$ of $O_{U}$ containing $I_{y}$ for all $y \in F$ such that as a $k[G]$-module $H^{0}\left(O_{U} / J\right)$ is isomorphic to the direct sum of $\rho_{0} \oplus \sigma$ and non-special representations.

Proof. Let $\rho \in \operatorname{Irr}(G)$ be special and non-trivial. If $y \in E(\rho)$, then $\alpha_{y}$ in (5.1.7) induces a homomorphism

$$
\operatorname{Ext}^{1}\left(O_{Z_{y}}, O_{0} \otimes_{k} \sigma\right) \rightarrow \operatorname{Ext}^{1}\left(\left(O_{U} \otimes_{k} \rho\right) / J(\rho), O_{0} \otimes_{k} \sigma\right)
$$

Suppose $W(\sigma) \subset \operatorname{Gen}\left(I_{y}\right)$. For a non-trivial element of $\operatorname{Ext}^{1}\left(O_{Z_{y}}, O_{0} \otimes_{k} \sigma\right) \neq$ 0 , there is a non-trivial extension $\left(\operatorname{ext}_{\sigma}\right)$ of Section 4.2:

$$
0 \rightarrow W(\sigma) \simeq I_{y} / J_{\sigma} \rightarrow O_{U} / J_{\sigma} \rightarrow O_{Z_{y}} \simeq O_{U} / I_{y} \rightarrow 0
$$

for some ideal $J_{\sigma}$ of $O_{U}$ such that $\mathfrak{m} I_{y} \subset J_{\sigma} \subset I_{y}$. The pullback of (6.2.2) by $O_{U} V(\rho) \hookrightarrow O_{Z_{y}}$ is given by

$$
0 \rightarrow W(\sigma) \simeq I_{y} / J_{\sigma} \rightarrow O_{U} \tilde{V}(\rho) / J_{\sigma} \rightarrow O_{U} V(\rho) \rightarrow 0 .
$$

Then we see, in the same manner as in Subsection 4.3, that $W(\sigma) \subset$ $\left(\mathfrak{m} \widetilde{V}(\rho)+\mathfrak{m} I_{y}\right) / \mathfrak{m} I_{y}$ if and only if (6.2.3) does not split if and only if (6.2.1) is non-zero. Since $O_{Z_{y}} \simeq \Phi_{O_{\mathcal{Z}}}\left(O_{y}\right)$ and $\left(O_{U} \otimes_{k} \rho\right) / J(\rho) \simeq \Phi_{O_{\mathcal{Z}}}\left(O_{E(\rho)}(-1)\right)$, (6.2.1) is isomorphic to

$$
\operatorname{Ext}^{1}\left(O_{y}, \Phi_{O_{\mathcal{Z}}}^{*}\left(O_{0} \otimes_{k} \sigma\right)\right) \rightarrow \operatorname{Ext}^{1}\left(O_{E(\rho)}(-1), \Phi_{O_{\mathcal{Z}}}^{*}\left(O_{0} \otimes_{k} \sigma\right)\right)
$$

induced by $O_{E(\rho)}(-1) \rightarrow O_{y}$. If $\sigma \neq \rho_{0}$, then by Corollary 3.19, (6.2.4) is

$$
\operatorname{Ext}^{1}\left(O_{y}, O_{E(\sigma)}(-1)\right) \rightarrow \operatorname{Ext}^{1}\left(O_{E(\rho)}(-1), O_{E(\sigma)}(-1)\right),
$$

which is non-zero if and only if $y \in E(\rho) \cap E(\sigma)$. Thus (1) is proved.

If $\sigma=\rho_{0}$, then (6.2.4) is isomorphic to

$$
\operatorname{Ext}^{2}\left(O_{y}, \omega_{F}\right) \rightarrow \operatorname{Ext}^{2}\left(O_{E(\rho)}(-1), \omega_{F}\right)
$$

Since $\omega_{F} \simeq O_{F}(F) \otimes_{O_{Y}} K_{Y}$, the Serre dual of (6.2.5) is

$$
\operatorname{Hom}\left(O_{F}(F), O_{E(\rho)}(-1)\right) \rightarrow \operatorname{Hom}\left(O_{F}(F), O_{y}\right),
$$

which is non-zero if and only if $F E(\rho)<0$. This proves (2).

Next we shall prove (3). Suppose $\rho=\rho_{0}$ and $y \in F$. Since $I_{y}$ is generated by special representations by Theorem 3.6, $I_{y}$ is contained in $J\left(\rho_{0}\right)$ and $\operatorname{Hom}\left(O_{Z_{y}}, O_{U} / J\left(\rho_{0}\right)\right)$ is a one-dimensional vector space generated by the natural surjection, which is denoted by $\psi$. 
Now suppose $y \in E(\sigma)$. Consider the cup product

$$
\begin{array}{r}
\operatorname{Hom}\left(O_{Z_{y}}, O_{U} / J\left(\rho_{0}\right)\right) \otimes_{k} \operatorname{Ext}^{1}\left(O_{U} / J\left(\rho_{0}\right), O_{0} \otimes_{k} \sigma\right) \\
\rightarrow \operatorname{Ext}^{1}\left(O_{Z_{y}}, O_{0} \otimes_{k} \sigma\right) .
\end{array}
$$

We show that $W(\sigma) \not \subset \mathfrak{m} J\left(\rho_{0}\right) / \mathfrak{m} I_{y}$ holds if and only if (6.2.6) is a nonzero map. Notice that $W(\sigma) \not \subset \mathfrak{m} J\left(\rho_{0}\right) / \mathfrak{m} I_{y}$ holds if and only if the composite

$$
W(\sigma) \hookrightarrow I_{y} / \mathfrak{m} I_{y} \rightarrow J\left(\rho_{0}\right) / \mathfrak{m} J\left(\rho_{0}\right)
$$

is injective. It is equivalent to the existence of an ideal $J^{\prime}$ with $\mathfrak{m} J\left(\rho_{0}\right) \subset$ $J^{\prime} \subset J\left(\rho_{0}\right)$ such that $W(\sigma) \rightarrow J\left(\rho_{0}\right) / J^{\prime}$ is an isomorphism, and therefore to the existence of a commutative diagram of the following form:

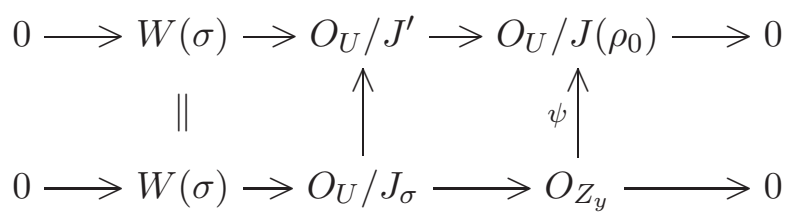

where the second row corresponds to the generator of $\operatorname{Ext}^{1}\left(O_{Z_{y}}, O_{0} \otimes_{k} \sigma\right)$. Hence the condition $W(\sigma) \not \subset \mathfrak{m} J\left(\rho_{0}\right) / \mathfrak{m} I_{y}$ is equivalent to the non-vanishing of $(6.2 .6)$.

Now we claim that (6.2.6) is isomorphic to

$$
\begin{aligned}
\operatorname{Ext}^{1}\left(O_{y}, \omega_{F}\right) & \otimes_{k} \operatorname{Hom}\left(\omega_{F}, O_{E(\sigma)}(-1)\right) \\
& \rightarrow \operatorname{Ext}^{1}\left(O_{y}, O_{E(\sigma)}(-1)\right) .
\end{aligned}
$$

By Lemma 5.10, $\Phi_{O \mathcal{Z}}^{(-1)}\left(\omega_{F}[1]\right)$ consists of non-special representations. Hence by Corollary $3.19 \Phi_{O_{\mathcal{Z}}}^{*}\left(\Phi_{O_{\mathcal{Z}}}^{(-1)}\left(\omega_{F}[1]\right)\right)=0$. This implies

$$
\Phi_{O_{\mathcal{Z}}}^{*}\left(O_{U} / J\left(\rho_{0}\right)\right) \simeq \Phi_{O_{\mathcal{Z}}}^{*}\left(\Phi_{O_{\mathcal{Z}}}^{0}\left(\omega_{F}[1]\right)\right) \simeq \Phi_{O_{\mathcal{Z}}}^{*}\left(\Phi_{O_{\mathcal{Z}}}\left(\omega_{F}[1]\right)\right) \simeq \omega_{F}[1]
$$

and therefore

$$
\operatorname{Hom}\left(O_{Z_{y}}, O_{U} / J\left(\rho_{0}\right)\right) \simeq \operatorname{Hom}\left(O_{y}, \Phi_{O_{Z}}^{*}\left(O_{U} / J\left(\rho_{0}\right)\right) \simeq \operatorname{Ext}^{1}\left(O_{y}, \omega_{F}\right) .\right.
$$

Next by the proof of Proposition 5.11, the following is also true:

$$
\operatorname{Ext}^{1}\left(O_{U} / J\left(\rho_{0}\right), O_{0} \otimes_{k} \sigma\right) \simeq \operatorname{Hom}\left(\omega_{F}, O_{E(\sigma)}(-1)\right) .
$$

We also have by Corollary 3.19

$$
\operatorname{Ext}^{1}\left(O_{Z_{y}}, O_{0} \otimes_{k} \sigma\right) \simeq \operatorname{Ext}^{1}\left(\Phi_{O_{\mathcal{Z}}}\left(O_{y}\right), O_{0} \otimes_{k} \sigma\right) \simeq \operatorname{Ext}^{1}\left(O_{y}, O_{E(\sigma)}(-1)\right) .
$$

Thus (6.2.6) is isomorphic to (6.2.7).

In $(6.2 .7)$, we see

$$
\operatorname{dim} \operatorname{Hom}\left(\omega_{F}, O_{E(\sigma)}(-1)\right)=a_{\rho_{0} \sigma}
$$

and hence if (6.2.7) is non-zero, then $a_{\rho_{0} \sigma} \geq 1$ and $y \in E(\sigma)$. Especially, if $a_{\rho_{0} \sigma}=0$, then we have $W(\sigma) \subset \mathfrak{m} J\left(\rho_{0}\right) / \mathfrak{m} I_{y}$ for any $y \in E(\sigma)$. 
Now let us prove $a_{\rho_{0} \sigma} \leq 2$. Since $F$ is the fundamental divisor, we have $(F-E(\sigma)) E(\sigma) \geq 0$, with equality holding if and only if $F=E(\sigma)$. Hence

$$
\begin{aligned}
a_{\rho_{0} \sigma}=-\left(K_{Y}+F\right) E(\sigma) & =-\left(\left(K_{Y}+E(\sigma)\right) E(\sigma)+(F-E(\sigma)) E(\sigma)\right) \\
& =-(-2+(F-E(\sigma)) E(\sigma)) \leq 2 .
\end{aligned}
$$

This implies that $a_{\rho_{0} \sigma} \leq 2$, and equality holds if and only if $F=E(\sigma)$. Moreover if $F=E(\sigma)$, then it is easy to see that $G$ is a cyclic group $\left\langle\frac{1}{n}(1,1)\right\rangle$ where $E(\sigma)^{2}=-n, \operatorname{dim} \sigma=1$ for any $\sigma \in \operatorname{Irr}(X)$. In this case, one can prove that (6.2.7) is non-zero, so that $W(\sigma) \not \subset \mathfrak{m} J\left(\rho_{0}\right) / \mathfrak{m} I_{y}$ for every $y \in E(\sigma)$.

It remains to consider the case $a_{\rho_{0} \sigma}=1$. Then

$$
\operatorname{Hom}\left(\omega_{F}, O_{E(\sigma)}(-1)\right) \simeq H^{0}\left(O_{E(\sigma)}\right) \simeq k .
$$

Therefore by the exact sequences

$$
\begin{aligned}
0 \rightarrow K_{Y} & \rightarrow K_{Y}(F) \rightarrow \omega_{F} \rightarrow 0 \\
0 \rightarrow K_{Y}(F-E(\sigma)) & \rightarrow K_{Y}(F) \rightarrow O_{E(\sigma)}(-1) \rightarrow 0,
\end{aligned}
$$

we see that (6.2.7) is equivalent to

$$
\operatorname{Ext}^{2}\left(O_{y}, K_{Y}\right) \rightarrow \operatorname{Ext}^{2}\left(O_{y}, K_{Y}(F-E(\sigma))\right)
$$

which is isomorphic to

$$
\operatorname{Ext}^{0}\left(O_{Y}, O_{y}\right)^{\vee} \rightarrow \operatorname{Ext}^{0}\left(O_{Y}(F-E(\sigma)), O_{y}\right)^{\vee}
$$

induced by the inclusion $O_{Y} \hookrightarrow O_{Y}(F-E(\sigma))$.

If $\operatorname{dim} \sigma=1$, then the coefficient of $E(\sigma)$ in $F$ is 1 and (6.2.8) is nonzero if and only if $y \in E(\sigma) \backslash(F-E(\sigma))$. Therefore, $W(\sigma) \not \subset \mathfrak{m} J\left(\rho_{0}\right) / \mathfrak{m} I_{y}$ holds for $y \in E(\sigma) \backslash(F-E(\sigma))$ and $W(\sigma) \subset \mathfrak{m} J\left(\rho_{0}\right) / \mathfrak{m} I_{y}$ holds for $y \in$ $E(\sigma) \cap(F-E(\sigma))$.

Finally suppose $\operatorname{dim} \sigma>1$. Then $G$ is not a cyclic group and hence $a_{\rho_{0} \sigma} \neq 0$ implies $a_{\rho_{0} \sigma}=1$. In this case, (6.2.8) is zero since the coefficient of $E(\sigma)$ in $F$ is greater than 1 and therefore (6.2.6) is also zero. Let $J \subset J\left(\rho_{0}\right)$ be the ideal with $J\left(\rho_{0}\right) / J=O_{0} \otimes_{k} \sigma^{\oplus a_{\rho_{0} \sigma}}=O_{0} \otimes_{k} \sigma$. Then the vanishing of (6.2.6) implies that $\psi: O_{Z_{y}} \rightarrow O_{U} / J\left(\rho_{0}\right)$ factors through $O_{U} / J$, which means $I_{y} \subset J$.

6.3. The integer $a_{\rho \rho_{0}}$. Theorem 6.7 (2) includes no information about $a_{\rho \rho_{0}}$, the number of arrows from $\rho$ to $\rho_{0}$ of the special McKay quiver. In order to explicitly describe it in terms of $G$-clusters, we consider Theorem 6.7 (2) for all $y \in E(\rho)$ simultaneously.

For a scheme $S$ of finite type over $k$, consider the integral functor

$$
\Phi_{O_{S} \otimes_{k} O_{\mathcal{Z}}}: D^{b}(S \times Y) \rightarrow D_{G}^{b}(S \times U)
$$

with kernel object $O_{S} \otimes_{k} O_{\mathcal{Z}}=\pi_{Y U}^{*}\left(O_{\mathcal{Z}}\right) \in D_{G}^{b}(S \times Y \times U)$, where $\pi_{Y U}$ is the projection to $Y \times U$. To be more precise, $\Phi_{O_{S} \otimes_{k} O_{\mathcal{Z}}}$ is defined as

$$
\Phi_{O_{S} \otimes_{k} O_{\mathcal{Z}}}(-)=\mathbf{R}\left(\pi_{S U}\right)_{*}\left(\mathbf{L} \pi_{S Y}^{*}(-) \stackrel{\mathbf{L}}{\otimes_{O S \times Y \times U}} \mathbf{L} \pi_{Y U}^{*}\left(O_{\mathcal{Z}}\right)\right) .
$$


Its right adjoint $\Phi_{O_{Y} \otimes_{k} O_{Z}}^{*}$ is also an integral functor whose kernel object is $O_{S} \otimes_{k}\left(K_{Y} \otimes_{O_{Y}} O_{\mathcal{Z}}^{\vee}[2]\right)$, the pull back of the kernel object of $\Phi_{O_{\mathcal{Z}}}^{*}$.

In parallel with $\Phi_{O_{\mathcal{Z}}}^{*} \circ \Phi_{O_{\mathcal{Z}}} \simeq I_{D^{b}(Y)}$, we have

$$
\Phi_{O_{Y} \otimes_{k} O_{\mathcal{Z}}}^{*} \circ \Phi_{O_{Y} \otimes_{k} O_{\mathcal{Z}}} \simeq I_{D^{b}(S \times Y)} .
$$

Moreover, the adjointness is refined as follows:

$$
\begin{aligned}
& \left(\mathbf{R}\left(\pi_{S}\right)_{*} \mathbf{R H o m}_{O_{S \times U}}\left(\Phi_{O_{Y} \otimes_{k} O_{\mathcal{Z}}}(\alpha), \beta\right)\right)^{G} \\
& \simeq \mathbf{R}\left(\pi_{S}\right)_{*} \mathbf{R} \text { Hom }_{O_{S \times Y}}\left(\alpha, \Phi_{O_{Y} \otimes_{k} O_{\mathcal{Z}}}^{*}(\beta)\right) .
\end{aligned}
$$

For a non-trivial special representation $\rho$, the $k[G]$-submodule $V(\rho) \subset O_{Z_{y}}$ depends on $y \in E(\rho)$ and is denoted by $V_{y}(\rho)$ in the sequel.

Lemma 6.8. There is an $O_{E(\rho)}$-submodule $\mathcal{V}(\rho) \subset O_{E(\rho)} \otimes_{O_{Y}} O_{\mathcal{Z}}$ (which is not necessarily an $O_{E(\rho) \times U}$-submodule) such that

$$
\mathcal{V}(\rho) \otimes_{O_{E(\rho)}} O_{y}=V_{y}(\rho)
$$

for $y \in E(\rho)$ and

$$
\mathcal{V}(\rho) \simeq O_{E(\rho)}(1) \otimes \rho .
$$

Proof. Let $E=E(\rho)$. We define $\mathcal{V}(\rho)$ as the image of the evaluation map

$$
\begin{aligned}
\left(\pi_{E}\right)_{*} \mathcal{H o m}_{O_{E \times U}}\left(O_{E} \otimes_{k}\left(O_{U} \otimes_{k} \rho / J(\rho)\right), O_{E \otimes_{O_{Y}} O_{\mathcal{Z}}}\right)^{G} & \otimes_{O_{E}}\left(O_{E} \otimes_{k} \rho\right) \\
& \rightarrow O_{E} \otimes_{O_{Y}} O_{\mathcal{Z}}
\end{aligned}
$$

of $O_{E}$-modules, where $O_{E} \otimes_{k} \rho \subset O_{E} \otimes_{k}\left(O_{U} \otimes_{k} \rho / J(\rho)\right)$ is the tensor product of $O_{E}$ with the constant term $\rho \subset O_{U} \otimes_{k} \rho / J(\rho)$. Taking the 0-th cohomology sheaves in (6.3.2) for $S=E$, we obtain

$$
\begin{aligned}
\left(\pi_{E}\right)_{*} \mathcal{H} \operatorname{mom}_{O_{E \times U}} & \left(O_{E} \otimes_{k}\left(O_{U} \otimes_{k} \rho / J(\rho), O_{E} \otimes_{O_{Y}} O_{\mathcal{Z}}\right)^{G}\right. \\
& \simeq\left(\pi_{E}\right)_{*} \mathcal{H} \operatorname{mom}_{O_{E \times Y}}\left(O_{E} \otimes_{k} O_{E}(-1), O_{\Delta_{E}}\right) \simeq O_{E}(1) .
\end{aligned}
$$

Therefore $\mathcal{V}(\rho)$ is the image of a non-trivial map

$$
O_{E}(1) \otimes_{k} \rho \rightarrow O_{E} \otimes_{O_{Y}} O_{\mathcal{Z}}
$$

which must be injective. Moreover, by the isomorphisms

$$
O_{E} \otimes_{O_{Y}} O_{\mathcal{Z}} \cong \bigoplus_{\rho} O_{E} \otimes_{O_{Y}} \mathcal{M}_{\rho} \otimes_{k} \rho
$$

and $O_{E} \otimes_{O_{Y}} \mathcal{M}_{\rho} \cong O_{E}^{\operatorname{dim} \rho-1} \oplus O_{E}(1)$, the cokernel of (6.3.3) is flat over $E$. Therefore, $\mathcal{V}(\rho) \otimes_{O_{E(\rho)}} O_{y} \rightarrow O_{Z_{y}}$ is also injective and this yields $\mathcal{V}(\rho) \otimes_{O_{E(\rho)}}$ $O_{y}=V_{y}(\rho)$.

By this lemma there is a map

$$
\alpha: O_{E(\rho)}(1) \otimes_{k} O_{U} \otimes_{k} \rho \rightarrow O_{E(\rho)} \otimes_{O_{Y}} O_{\mathcal{Z}}
$$

of sheaves on $E(\rho) \times U$ such that its restriction to the fiber over $y \in E(\rho)$ coincides with $\alpha_{y}$ in (5.1.7). 
Recall we have

$$
\mathcal{I} /\left(\mathfrak{m} \mathcal{I}+I_{F}\right) \simeq\left(\sum_{\tau \neq \rho_{0}} O_{E(\tau)}(-1) \otimes_{k} \tau\right) \oplus O_{F}(-F) \otimes_{k} \rho_{0}
$$

by Theorem 3.13 and take the ideal $\mathcal{I}_{\rho, \rho_{o}}$ with $\mathfrak{m} \mathcal{I}+I_{F} \subset \mathcal{I}_{\rho, \rho_{0}} \subset \mathcal{I}$ such that

$$
\mathcal{I} / \mathcal{I}_{\rho, \rho_{0}}=\mathcal{W}\left(\rho_{0}\right):=O_{E(\rho)}(-F) \otimes_{k} \rho_{0} .
$$

Then, by $H^{1}\left(O_{E(\rho)}(-1) \otimes_{O_{Y}} \mathcal{I} / \mathcal{I}_{\rho, \rho_{0}}\right)=0, \alpha$ can be lifted to

$$
\tilde{\alpha}: O_{E(\rho)}(1) \otimes_{k} O_{U} \otimes_{k} \rho \rightarrow O_{E(\rho)} \otimes_{O_{Y}} O_{Y \times U} / \mathcal{I}_{\rho, \rho_{0}} .
$$

Since $\alpha$ factors through $O_{E(\rho)}(1) \otimes_{k}\left(O_{U} \otimes_{k} \rho / J(\rho)\right)$, $\tilde{\alpha}$ induces a map

$$
O_{E(\rho)}(1) \otimes_{k}(J(\rho) / \mathfrak{m} J(\rho))^{G} \rightarrow\left(\mathcal{I} / \mathcal{I}_{\rho, \rho_{0}}\right)^{G}
$$

which is isomorphic to

$$
O_{E(\rho)}(1)^{\oplus a_{\rho \rho} \rho_{0}} \rightarrow O_{E(\rho)}\left(a_{\rho \rho_{0}}\right)
$$

where $a_{\rho \rho_{0}}=-F E(\rho)$. By (6.3.4) we have $a_{\rho \rho_{0}}$ maps in

$$
\operatorname{Hom}\left(O_{E(\rho)}(1), O_{E(\rho)}\left(a_{\rho \rho_{0}}\right)\right)
$$

which corresponds to $a_{\rho \rho_{0}}$ arrows from $\rho$ to $\rho_{0}$. In fact, the following explains the $a_{\rho \rho_{0}}$ arrows in terms of $G$-clusters.

Proposition 6.9. The $a_{\rho \rho_{0}}$ maps given by (6.3.4) form a basis of

$$
\operatorname{Hom}\left(O_{E(\rho)}(1), O_{E(\rho)}\left(a_{\rho \rho_{0}}\right)\right) .
$$

Proof. Put $E=E(\rho)$. The $O_{E}$-dual of (6.3.4) is

$$
\begin{aligned}
\left(\pi_{E}\right)_{*} \mathcal{H o m}_{O_{E \times U}}\left(\left.\mathcal{I}\right|_{E \times U}, O_{E \times 0} \otimes_{k} \rho_{0}\right)^{G} & \\
& \rightarrow\left(\pi_{E}\right)_{*} \mathcal{H o m} O_{E \times U}\left(O_{E}(1) \otimes_{k} J(\rho), O_{E \times 0} \otimes_{k} \rho_{0}\right)^{G},
\end{aligned}
$$

which is a map of sheaves on $E \simeq E \times 0$. From the short exact sequence $0 \rightarrow O_{E}(1) \otimes_{k} J(\rho) \rightarrow O_{E}(1) \otimes_{k}\left(O_{U} \otimes_{k} \rho\right) \rightarrow O_{E}(1) \otimes_{k}\left(O_{U} \otimes_{k} \rho / J(\rho)\right) \rightarrow 0$ we derive an isomorphism

$$
\begin{aligned}
\mathcal{H o m}_{O_{E \times U}}\left(O_{E}(1) \otimes_{k} J(\rho), O_{E \times 0} \otimes_{k} \rho_{0}\right) \\
\simeq \mathcal{E} x t_{O_{E \times U}}^{1}\left(O_{E}(1) \otimes_{k}\left(O_{Y} \otimes_{k} \rho / J(\rho)\right), O_{E \times 0} \otimes_{k} \rho_{0}\right),
\end{aligned}
$$

while from the exact sequence

$$
\left.\left.0 \rightarrow \mathcal{I}\right|_{E \times U} \rightarrow O_{E \times U} \rightarrow O_{\mathcal{Z}}\right|_{E \times U} \rightarrow 0
$$

we derive an isomorphism

$$
\begin{aligned}
\mathcal{H o m}_{O_{E \times U}} & \left(\left.\mathcal{I}\right|_{E \times U}, O_{E \times 0} \otimes_{k} \rho_{0}\right) \\
& \simeq \mathcal{E} x t_{O_{E \times U}}^{1}\left(\left.O_{\mathcal{Z}}\right|_{E \times U}, O_{E \times 0} \otimes_{k} \rho_{0}\right) .
\end{aligned}
$$


Hence (6.3.5) is isomorphic to

$$
\begin{array}{rl}
\left(\pi_{E}\right)_{*} & \mathcal{E} x t_{O_{E \times U}}^{1}\left(\left.O_{\mathcal{Z}}\right|_{E \times U}, O_{E \times 0} \otimes_{k} \rho_{0}\right)^{G} \\
& \rightarrow\left(\pi_{E}\right)_{*} \mathcal{E} x t_{O_{E \times U}}^{1}\left(O_{E}(1) \otimes_{k}\left(O_{Y} \otimes_{k} \rho / J(\rho)\right), O_{E \times 0} \otimes_{k} \rho_{0}\right)^{G}
\end{array}
$$

(this is a family of maps (6.2.1) parametrized by $y \in E$ ). By (6.3.2), the map (6.3.6) is isomorphic to

$$
\begin{array}{rl}
\left(\pi_{E}\right)_{*} & \mathcal{E} x t_{O_{E \times Y}}^{2}\left(O_{\Delta_{E}}, O_{E} \otimes_{k} \omega_{F}\right) \\
& \rightarrow\left(\pi_{E}\right)_{*} \mathcal{E} x t_{O_{E \times Y}}^{2}\left(O_{E}(1) \otimes_{k} O_{E}(-1), O_{E} \otimes \omega_{F}\right)
\end{array}
$$

which is a family of maps (6.2.4). Taking the dual of (6.3.7), we see that (6.3.4) is isomorphic to

$$
\begin{aligned}
\left(\pi_{E}\right)_{*} \mathcal{H o m}_{O_{E \times Y}}\left(O_{E} \otimes_{k} \omega_{F}, O_{E}(1) \otimes_{k}\left(O_{E}(-1) \otimes_{O_{Y}} K_{Y}\right)\right. \\
\rightarrow\left(\pi_{E}\right)_{*} \mathcal{H} \operatorname{Hom}_{O_{E \times Y}}\left(O_{E} \otimes_{k} \omega_{F}, O_{\Delta_{E}} \otimes K_{Y}\right)
\end{aligned}
$$

which is determined by the restriction map

$$
\left.O_{E}(1) \otimes_{k} O_{E}(-1) \rightarrow\left(O_{E}(1) \otimes_{k} O_{E}(-1)\right)\right|_{\Delta_{E}} \simeq O_{E} .
$$

Since $\left.\omega_{F} \simeq K_{Y}(F)\right|_{F}$, (6.3.8) is isomorphic to the evaluation map

$$
\operatorname{Hom}\left(O_{F}(-1), O_{E}(-F)\right) \otimes_{k} O_{E}(1) \rightarrow O_{E}(-F)
$$

and we are done.

\section{EXAMPLES}

7.1. The cyclic group $G=\left\langle\frac{1}{12}(1,5)\right\rangle$. Consider the case of a cyclic group

$$
G=\left\langle\frac{1}{12}(1,5)\right\rangle=\left\langle\left(\begin{array}{cc}
\zeta_{12} & 0 \\
0 & \zeta_{12}^{5}
\end{array}\right)\right\rangle
$$

where $\zeta_{12}$ is the primitive 12 -th root of unity. Let $g=\operatorname{diag}\left(\zeta_{12}, \zeta_{12}^{5}\right)$ be a generator of $G$, and $\rho_{i} \in \operatorname{Irr}(G)=\operatorname{Hom}_{\mathbf{Z}}\left(G, \mathbf{G}_{m}\right)$ for $i(\bmod 12)$ such that $\rho_{i}(g)=\zeta_{12}^{-i}$. Let $S_{1}$ be the $G$-submodule spanned by $x$ and $y$. The group $G$ acts on $U$ diagonally so that $S_{1} \simeq \rho_{1} \oplus \rho_{5}$ as $k[G]$-modules, the $\rho_{1}$-part (resp. $\rho_{5}$-part) being spanned by $x$ (resp. $y$ ). The $k[G]$-module $k[x, y]$ is decomposed into irreducible pieces, each spanned by a single monomial. Associated to every monomial $x^{a} y^{b}$ in Figure 1, we have a representation $\rho_{i}$ with $i=a+5 b(\bmod 12)$ in the same Figure, which is quoted as $i$ instead of $\rho_{i}$ for short. Figure 1 can be seen as (a covering of) the McKay quiver, where the arrow $i \rightarrow i+1(\bmod 12)($ resp. $i \rightarrow i+5(\bmod 12))$ corresponds to multiplication by $x$ (resp. $y$ ).

The special representations of $G$ are $\rho_{0}, \rho_{1}, \rho_{3}$ and $\rho_{5}$, which are determined by the continued fraction expansion of $\frac{12}{5}$ ([33]). The modules $O_{U} \otimes_{k} \rho_{i} / J\left(\rho_{i}\right)$ and the generators of $J\left(\rho_{i}\right)$ for the special representations are given in Figure 2. The framed monomials in those Figures form a basis of the vector space $O_{U} \otimes_{k} \rho_{i} / J\left(\rho_{i}\right)$ and the monomials outside the frame are minimal generators of $J\left(\rho_{i}\right)$. 


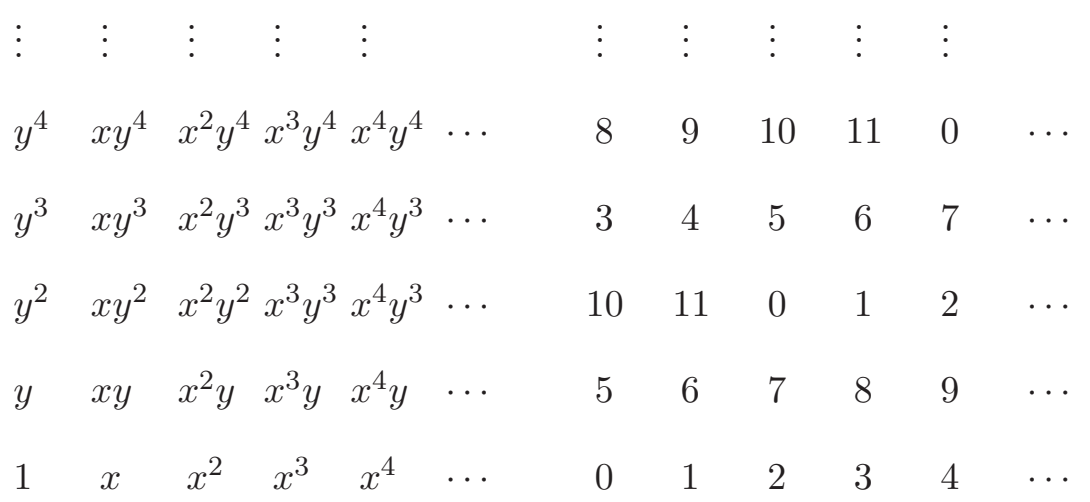

FIGURE 1. monomials and weights

If we look at Figure 2, then we find three paths in the covering of the McKay quiver from the vertex $\rho_{1}$, the generator of $O_{U} \otimes_{k} \rho_{1}$, to two $\rho_{0}$ 's outside of $O_{U} \otimes_{k} \rho_{i} / J\left(\rho_{i}\right)$. The two paths from $\rho_{1}$ to $\rho_{0}$ in the McKay quiver corresponds to $\phi_{1,0}^{1}$ and $\phi_{1,0}^{2}$ in Subsection 6.1, which determine the two arrows from $\rho_{1}$ to $\rho_{0}$ in the special McKay quiver $Q_{X}$ associated to the reconstruction algebra. From Figure 2 we can read the matrix $\left(a_{\rho_{i} \rho_{j}}\right)$ as follows:

$$
\left(a_{\rho_{i} \rho_{j}}\right)_{i, j=0,1,3,5}=\left(\begin{array}{cccc}
0 & 1 & 0 & 1 \\
2 & 0 & 1 & 0 \\
0 & 1 & 0 & 1 \\
2 & 0 & 1 & 0
\end{array}\right),
$$

which determines the special McKay quiver $Q_{X}$ in an obvious way.

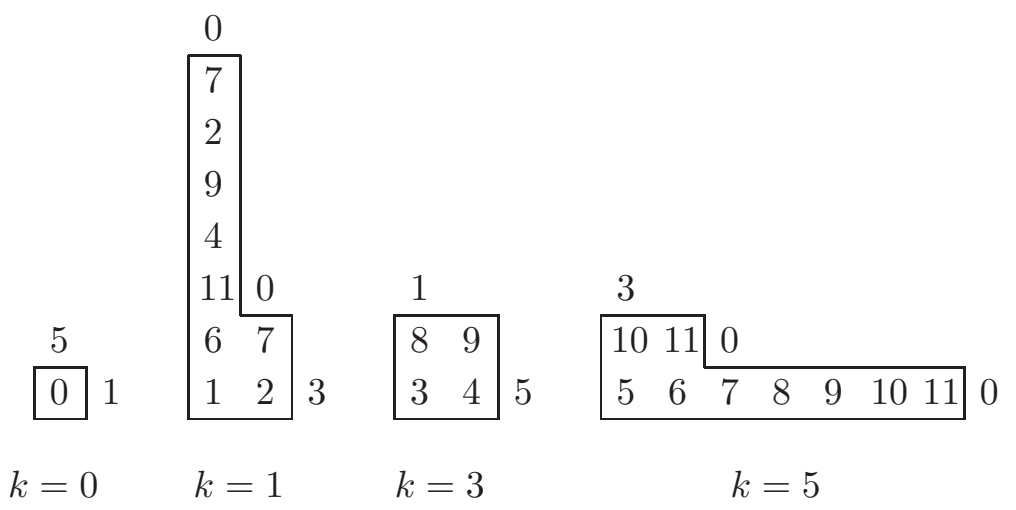

FiguRE 2. $O_{U} \otimes_{k} \rho_{k} / J\left(\rho_{k}\right)$

Now we explain Theorem 6.7 in the case of this example. Suppose $E\left(\rho_{3}\right) \cap E\left(\rho_{5}\right)=\{y\}$. The structure sheaf $O_{Z_{y}}$ of the $G$-cluster $Z_{y}$ has a $k$-basis consisting of 12 monomials framed in Figure 3. We note $O_{Z_{y}} \simeq$ 


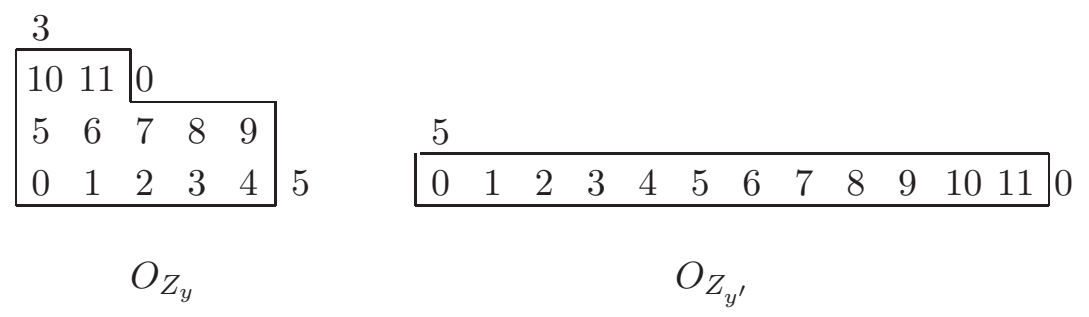

FiguRE 3. $O_{Z_{y}}$ and $O_{Z_{y^{\prime}}}$

$k[G] \simeq \oplus_{i=0}^{11} \rho_{i}$. Let $V\left(\rho_{i}\right)$ be the $\rho_{i}$-part of $O_{Z_{y}}$ for $i=3,5$. By a direct computation, we see that the ideal $O_{U} V\left(\rho_{i}\right)$ of $O_{Z_{y}}$ contains no other special representation for $i=3,5$ as in Theorem 5.7. Moreover, $W\left(\rho_{3}\right), W\left(\rho_{0}\right)$ and $W\left(\rho_{5}\right)$ are located at the corners of the complement of the framed part in Figure 3. Then it is easy to observe that $\left(\widetilde{V}\left(\rho_{3}\right) O_{U}+\mathfrak{m} I_{y}\right) / \mathfrak{m} I_{y}$ contains $W\left(\rho_{5}\right)$ and $\left(\widetilde{V}\left(\rho_{5}\right) O_{U}+\mathfrak{m} I_{y}\right) / \mathfrak{m} I_{y}$ contains $W\left(\rho_{3}\right)$ as in Theorem 6.7 (1).

Since $y \in E\left(\rho_{5}\right)$, we have $\left(\widetilde{V}\left(\rho_{5}\right) O_{U}+\mathfrak{m} I_{y}\right) / \mathfrak{m} I_{y}$ contains $W\left(\rho_{0}\right)$, which gives rise to one path of the special McKay quiver from $\rho_{5}$ to $\rho_{0}$, that is, an $O_{U}-G$-homomorphism from from $O_{U} \otimes_{k} \rho_{5}$ to $O_{U} \otimes_{k} \rho_{0}$. The point $y^{\prime}$ corresponding to $Z_{y^{\prime}}$ in Figure 3 is another point of $E\left(\rho_{5}\right)$, which gives rise to another path from $\rho_{5}$ to $\rho_{0}$. These two paths are precisely the two paths in Figure 2 corresponding to $\phi_{5,0}^{1}$ and $\phi_{5,0}^{2}$. This explains Theorem 6.7 (2) and Proposition 6.9.

Finally, Figure 2 shows that there is an arrow from $\rho_{0}$ to $\rho_{1}$ (resp. from $\rho_{0}$ to $\left.\rho_{5}\right)$. No arrow from $\rho_{0}$ to $\rho_{5}$ appears at $y^{\prime} \in E\left(\rho_{5}\right)$, while an arrow from $\rho_{0}$ to $\rho_{5}$ does appear at $y \in E\left(\rho_{5}\right)$, as in the third case in Theorem 6.7 (3a). The case $\sigma=\rho_{1}$ is similar. If $\sigma \neq \rho_{1}, \rho_{5}$, then the first case of Theorem 6.7 (3a) occurs.

7.2. The binary dihedral case $D_{5}$. Let $S=k[x, y]$ and $U=\mathbf{A}_{k}^{2}=$ Spec $S$. The simple singularity $D_{5}$ is the quotient singularity of $U$ by the binary dihedral group $G:=\mathbf{D}_{3}$ of order 12 , which is generated by $\sigma$ and $\tau$ :

$$
\sigma=\left(\begin{array}{cc}
\epsilon & 0 \\
0 & \epsilon^{-1}
\end{array}\right), \quad \tau=\left(\begin{array}{cc}
0 & 1 \\
-1 & 0
\end{array}\right)
$$

where $\epsilon:=e^{2 \pi i / 6}$. We have $\sigma^{6}=\tau^{4}=1, \sigma^{3}=\tau^{2}$ and $\tau \sigma \tau^{-1}=\sigma^{-1}$. The group $G$ acts on $U$ from the right by $(x, y) \mapsto(x, y) g$ for $g \in G$, hence, $\tau$ acts on the ring $S$ is defined to be $\tau(x)=-y$ and $\tau(y)=x$. The ring of $G$-invariants in $S$ is generated by three elements

$$
A_{6}:=x^{6}+y^{6}, A_{8}:=x y\left(x^{6}-y^{6}\right), A_{4}:=x^{2} y^{2} .
$$

The quotient $U / G$ is isomorphic to the hypersurface $4 A_{4}^{4}+A_{8}^{2}-A_{4} A_{6}^{2}=0$. 
We quote some of the data from [21, p. 200]. By [21, Table 8, p. 200], The list of possible generators of $\operatorname{Gen}\left(I_{y}\right)(y \in E)$ is given by

$$
\begin{aligned}
V_{4}\left(\rho_{0}\right) \oplus V_{6}\left(\rho_{0}\right) & =\left\{x^{2} y^{2}\right\} \oplus\left\{x^{6}+y^{6}\right\}, \\
V_{2}\left(\rho_{1}\right) \oplus V_{6}\left(\rho_{1}\right) & =\{x y\} \oplus\left\{x^{6}-y^{6}\right\}, \\
V_{3}\left(\rho_{2}\right) \oplus V_{5}\left(\rho_{2}\right) & =\left\{x^{2} y,-x y^{2}\right\} \oplus\left\{y^{5},-x^{5}\right\}, \\
V_{4}\left(\rho_{3}\right) & =\left\{y^{4}, x^{4}\right\} \oplus\left\{x^{3} y,-x y^{3}\right\}, \\
V_{3}\left(\rho_{4}\right) \oplus V_{5}\left(\rho_{4}\right) & =\left\{x^{3}+i y^{3}\right\} \oplus\left\{x y\left(x^{3}-i y^{3}\right)\right\}, \\
V_{3}\left(\rho_{5}\right) \oplus V_{5}\left(\rho_{5}\right) & =\left\{x^{3}-i y^{3}\right\} \oplus\left\{x y\left(x^{3}+i y^{3}\right)\right\} .
\end{aligned}
$$

Let $S_{1}$ be the space spanned by $x$ and $y$. Then multiplication by any element of $S_{1}$ defines an endomorphism of Coinv $\left(D_{5}\right)$, which induces a homomorphism from an irreducible factor of Coinv $\left(D_{5}\right)$ to another irreducible factor. Let $y \in F \subset G$-Hilb $(U)$. Let $\rho \simeq V(\rho) \subset \operatorname{Soc}\left(O_{Z_{y}}\right)$ and $\rho^{\prime} \simeq W\left(\rho^{\prime}\right) \subset \operatorname{Gen}\left(I_{y}\right)$. If $W\left(\rho^{\prime}\right) \subset S_{1} V(\rho)$, we draw a directed arrow from $V(\rho)$ to $W\left(\rho^{\prime}\right)$. As was explained in [21, p. 201], if we consider all pairs $\rho, \sigma \in \operatorname{Irr}(G)$ nontrivial, this gives the Dynkin diagram $D_{5}$ with pairs of directed arrows in opposite direction as part of Figure 4. See [21, p. 201] and $[25]$ for details.

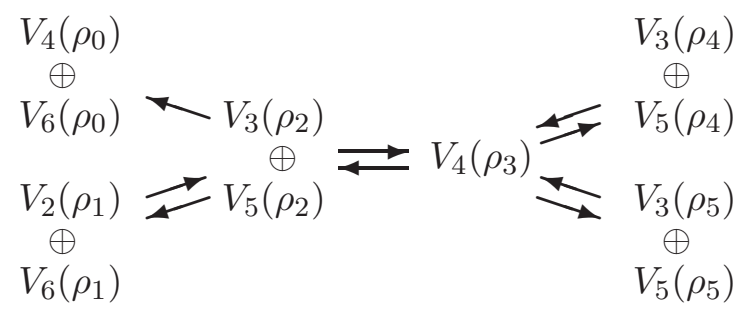

Figure 4. Directed arrows of $\widetilde{D}_{5}$

In what follows, we explain what happens at the vertex $\rho_{0}$ of the extended Dynkin diagram $\widetilde{D}_{5}$. The result looks somewhat novel. It is slightly different from $\left[25\right.$, p. 277 fifth line from below; p. 278, Fig. $\left.6^{8}\right]$.

Let $V^{\sharp}\left(\rho_{0}\right)=V_{4}\left(\rho_{0}\right) \oplus V_{6}\left(\rho_{0}\right)$, and $V^{\sharp}\left(\rho_{2}\right)=V_{4}\left(\rho_{0}\right) \oplus V_{6}\left(\rho_{0}\right)$. Recall $E\left(\rho_{2}\right)=\mathbf{P}\left(V^{\sharp}\left(\rho_{2}\right)\right)$. Let $W\left(\simeq \rho_{2}\right) \subset V^{\sharp}\left(\rho_{2}\right)$, and $x=I_{x}:=I(W)$ any point of $E\left(\rho_{2}\right)$. Then

$$
\begin{aligned}
\operatorname{Soc}\left(O_{Z_{x}}\right)\left[\rho_{2}\right] & \simeq V_{3}\left(\rho_{2}\right) \oplus V_{5}\left(\rho_{2}\right) / W, \\
\left(S_{1} \cdot\left(\operatorname{Soc}\left(O_{Z_{x}}\right)\left[\rho_{2}\right]\right)\right)\left[\rho_{0}\right] & \simeq\left(S_{1} V_{3}\left(\rho_{2}\right) \oplus S_{1} V_{5}\left(\rho_{2}\right)\right)\left[\rho_{0}\right] /\left(S_{1} W\right)\left[\rho_{0}\right], \\
& \simeq V^{\sharp} / V^{\sharp} \cap\left(S_{1} W\right) \simeq \operatorname{Gen}\left(I_{x}\right)\left[\rho_{0}\right],
\end{aligned}
$$

\footnotetext{
${ }^{8}$ The arrow from $V_{4}\left(\rho_{0}\right)$ to $V_{3}\left(\rho_{2}\right)$ in [25, Fig. 6] has to be reversed. See [25, Fig. 14].
} 
where $\operatorname{Soc}\left(O_{Z_{x}}\right)=[I(W): \mathfrak{m}] / \mathfrak{m} I(W)$, and $S_{1} \operatorname{Soc}\left(O_{Z_{x}}\right)=S_{1}\left(\left[I_{x}: \mathfrak{m}\right] / I_{x}\right) \subset$ $I_{x} / \mathfrak{m} I_{x}=\operatorname{Gen}\left(I_{x}\right)$. This defines an isomorphism from $E\left(\rho_{2}\right)$ to $\mathbf{P}\left(V^{\sharp}\left(\rho_{0}\right)\right)$ sending $W$ to $V^{\sharp} / V^{\sharp} \cap\left(S_{1} W\right)$. Note that there is one more arrow from $\rho_{0}$ to $\rho_{2}$ in the McKay quiver, which is understood as an arrow from $V_{0}\left(\rho_{0}\right)$ to $V_{1}\left(\rho_{2}\right)$ as in Theorem $6.7(3 \mathrm{~b})$.

Remark 7.1. For every other rational double singularity A similar structure of the coinvariant algebra is observed by using Subsection 4.2 and [21, Tables $7,10,13,17]$. See also $\left[25\right.$, p. 289, Fig. 14]. In the $D_{4}$ case, the subspace $V_{4}^{\prime}\left(\rho_{0}\right)=\left\{x^{2} y^{2}\right\}$ (resp. $V_{4}^{\prime \prime}\left(\rho_{0}\right)=\left\{x^{4}+y^{4}\right\}$ plays the same role as $V_{4}\left(\rho_{0}\right)$ (resp. $\left.V_{6}\left(\rho_{0}\right)\right)$ of $D_{5}$. In the $A_{n}$ case $(n \geq 2)$, there is an arrow $\rho_{1}$ to $\rho_{0}$ (resp. from $\rho_{n}$ to $\rho_{0}$ ) as above, while the other pairs are opposite.

Remark 7.2. In the $A_{1}$ case, there is only one arrow from $\rho_{1}$ to $\rho_{0}$. Indeed, let $S_{k}$ be the space of polynomials of degree $k(k=1,2)$. Then $E\left(\rho_{1}\right)=$ $\mathbf{P}\left(S_{1}\right)$. Let $x=I(W)$ be any point of $E\left(\rho_{1}\right)$. Then $I_{x}=O_{U} W$, $\operatorname{Soc}\left(O_{Z_{x}}\right)=$ $S_{1} / W$. Hence we have a morphism $f$ from $\mathbf{P}\left(S_{1}\right)$ to $\mathbf{P}\left(S_{2}\right)$ sending $W$ to $S_{2} / S_{1} W=S_{1}\left(S_{1} / W\right)$. This induces an isomorphism from $\mathbf{P}\left(S_{1}\right)$ to a nonsingular conic of $\mathbf{P}\left(S_{2}\right)$.

\section{REFERENCES}

[1] M. Artin, On isolated rational singularities of surfaces, Amer. Jour. Math., 88 (1966) 129-136.

[2] M. Artin, J.-L. Verdier, Reflexive modules over rational double points, Math. Ann., 270 (1985) 79-82.

[3] M. Auslander, Rational singularities and almost split sequences, Trans. Amer. Math. Soc. 293 (1986), no. 2, 511-531.

[4] A. I. Bondal, M. M. Kapranov, Representable functors, Serre functors, and reconstructions, Izv. Akad. Nauk SSSR Ser. Mat. 53 (1989), no. 6, 1183-1205, 1337

[5] T. Bridgeland, Equivalences of triangulated categories and Fourier-Mukai transforms, Bull. London Math. Soc. 31 (1999) 25-34.

[6] T. Bridgeland, A. King, M. Reid, The McKay correspondence as an equivalence of derived categories, Jour. Amer. Math. Soc., 14 (2001) 535-554.

[7] A. Craw, The special McKay correspondence as an equivalence of derived categories, Q. J. Math., 62, no. 3 (2011), 573-591.

[8] H. Esnault, Reflexive modules on quotient surface singularities, Jour. reine angew. Math., 362 (1985) 63-71.

[9] H. Esnault, H. Knörrer, Reflexive modules over rational double points, Math. Ann., 272 (1985) 545-548.

[10] A. Grothendieck, Éléments de géométrie algébrique, I,II,III,IV, Publ. Math. IHES, 4 (1960), 8 (1961), 11 (1961), 17 (1963), 20 (1964), 24 (1965), 28 (1966), 32 (1967).

[11] A. Grothendieck, Revêtements Etales et Groupe Fondamentale (SGA 1), Lect. Notes Math., 224, Springer-Verlag, 1971.

[12] A. Grothendieck, Local cohomology, Lecture Notes in Math., 41, Springer-Verlag, Berlin Heidelberg New York, 1967.

[13] G. Gonzalez-Sprinberg, J. Verdier, Construction géométrique de la correspondence de McKay, Ann. scient. Éc. Norm. Sup. 16 (1983) 409-449.

[14] R. Hartshorne, Residue and Duality, Lecture Notes in Mathematics, 20, Springer Verlag, Berlin Heidelberg New York, 1966. 
[15] R. Hartshorne, Algebraic Geometry, Graduate Texts in Mathematics, 52, Springer Verlag, Berlin Heidelberg New York, 1977.

[16] R. Hartshorne, Stable reflexive sheaves, Math. Ann., 254, (1980) 121-176.

[17] D. Huybrechts, Fourier-Mukai transforms in algebraic geometry, Oxford Mathematical Monographs, The Clarendon Press, Oxford Univ. Press, Oxford, 2006.

[18] A. Ishii, On the moduli of reflexive sheaves on a surface with rational double points, Math. Ann. 294 (1992) 125-150.

[19] A. Ishii, On the McKay correspondence for a finite small subgroup of GL(2, C), Jour. reine angew. Math., 549 (2002) 221-233.

[20] A. Ishii and K. Ueda, The special McKay correspondence and exceptional collections, Tohoku Math. J. (2) 67 (2015), no. 4, 585-609.

[21] Y. Ito and I. Nakamura, Hilbert schemes and simple singularities, New trends in algebraic geometry, Proceedings of European Math. Conference 1996, London Mathematical Society Lecture Note Series 264, Cambridge University Press (1999) 151-233.

[22] M. Kapranov, E. Vasserot, Kleinian singularities, derived categories and Hall algebras, Math. Ann., 316, (2000) 565-576.

[23] J. Lipman, Rational singularities, Publ. Math. Inst. Hautes. Etudes Sci. 36, (1969) 195-279.

[24] J. McKay, Graphs, singularities, and finite groups, in Santa Cruz, conference on finite groups (Santa Cruz, 1979), Proc. Symp. Pure Math., AMS 37, 1980, pp. 183-186.

[25] I. Nakamura, McKay correspondence, Centre de Recherches Mathématiques CRM Proceedings and Lecture Notes, 47, 2009, pp. 267-298.

[26] D. Quillen, Projective modules over polynomial ring, Inv. Math. 36 (1976) 167-171.

[27] A. Suslin. Doklady Akademii Nauk SSSR (in Russian) 229, (1976) 1063-1066; Translated in "Projective modules over polynomial rings are free", Soviet Mathematics 17, (1976) 1160-1164.

[28] E. Viehweg, Rational singularities of higher dimensional schemes, Proc. Amer. Math. Soc. 63 (1997) 6-8.

[29] M. Wemyss, The GL(2, C) McKay correspondence, Math. Ann., 350 (2011) 631-659

[30] , Reconstruction algebras of type A, Trans. Amer. Math. Soc. 363 (2011), no. $6,3101-3132$.

[31] Reconstruction algebras of type D (I), J. Algebra 356 (2012), 158-194.

[32] _ Reconstruction algebras of type D (II), Hokkaido Math. J. 42 (2013), 293329.

[33] J. Wunram, Reflexive modules on quotient surfaces singularities, Math. Ann., 279 (1988) 583-598.

Division of Mathematical and Information Sciences, Hiroshima University, 1-7-1 Kagamiyama, Higashi-Hiroshima, 739-8521, JaPAN

E-mail address: akira141@hiroshima-u.ac.jp

Department of Mathematics, Hokkaido University, Sapporo, 060-0810, Japan

E-mail address: nakamura@math.sci.hokudai.ac.jp 